\title{
Mean dynamics of transitional channel flow
}

\author{
J. ELSNAB ${ }^{1}$, J. KLEWICKI ${ }^{2,3} \dagger$, D. MAYNES AND T. AMEEL \\ ${ }^{1}$ Department of Mechanical Engineering, University of Utah, Salt Lake City, UT 84112, USA \\ ${ }^{2}$ Department of Mechanical Engineering, University of New Hampshire, Durham, NH 03824, USA \\ ${ }^{3}$ Department of Mechanical Engineering, University of Melbourne, Melbourne, VIC 3010, Australia \\ ${ }^{4}$ Department of Mechanical Engineering, Brigham Young University, Provo, UT 84602, USA
}

(Received 13 May 2010; revised 11 January 2011; accepted 7 March 2011;

first published online 3 May 2011)

The redistribution of mean momentum and vorticity, along with the mechanisms underlying these redistribution processes, is explored for post-laminar flow in fully developed, pressure driven, channel flow. These flows, generically referred to as transitional, include an instability stage and a nonlinear development stage. The central focus is on the nonlinear development stage. The present analyses use existing direct numerical simulation data sets, as well as recently reported high-resolution molecular tagging velocimetry measurements. Primary considerations stem from the emergence of the effects of turbulent inertia as represented by the Reynolds stress gradient in the mean differential statement of dynamics. The results describe the flow evolution following the formation of a non-zero Reynolds stress peak that is known to first arise near the critical layer of the most unstable disturbance. The positive and negative peaks in the Reynolds stress gradient profile are observed to undergo a relative movement toward both the wall and centreline for subsequent increases in Reynolds number. The Reynolds stress profiles are shown to almost immediately exhibit the same sequence of curvatures that exists in the fully turbulent regime. In the transitional regime, the outer inflection point in this profile physically indicates a localized zone within which the mean dynamics are dominated by inertia. These observations connect to recent theoretical findings for the fully turbulent regime, e.g. as described by Fife, Klewicki \& Wei (J. Discrete Continuous Dyn. Syst., vol. 24, 2009, p. 781) and Klewicki, Fife \& Wei (J. Fluid Mech., vol. 638, 2009, p. 73). In accord with momentum equation analyses at higher Reynolds number, the present observations provide evidence that a logarithmic mean velocity profile is most rapidly approximated on a sub-domain located between the zero in the Reynolds stress gradient (maximum in the Reynolds stress) and the outer region location of the maximal Reynolds stress gradient (inflection point in the Reynolds stress profile). Overall, the present findings provide evidence that the dynamical processes during the post-laminar regime and those operative in the high Reynolds number regime are connected and describable within a single theoretical framework.

Key words: boundary layer structure, turbulent boundary layers, turbulent transition

\section{Introduction}

Laminar to turbulent transition in pressure driven channel flow is marked by dramatic changes in the mean profiles of momentum and vorticity. 
Primary characteristics of post-laminar flow evolution are a rapid trend toward homogenization of the mean momentum field, in concert with a wallward redistribution of mean vorticity. Channel flow transition is also characterized by similarly rapid changes in the skin friction and pressure drop. The momentum and vorticity field redistribution processes initiated persist post-transition, and describing the rates at which these processes continue to evolve with increasing Reynolds number effectively constitutes the mean flow scaling problem in the turbulent regime. Of course, the dynamical mechanisms responsible for the redistribution processes following the initial instability stage are associated with the emergence and growth of nonlinearly correlated fluctuations. In the mean, these are mathematically represented by an additional force-like term in the relevant differential statement of Newton's second law. A central objective of the present study is to clarify how this mean effect of turbulent inertia combines with the already existent pressure and viscous forces to form a balance over the relevant Reynolds number range.

Herein we generically refer to the phenomena of interest as transitional. These phenomena, however, actually occur in two stages. The first is an instability stage relating to the onset and establishment of self-sustaining nonlinear mechanisms. The second is a nonlinear flow development stage in which the dynamics evolve to a state that is formally representative of high Reynolds number turbulence. Our focus is on the Reynolds number range starting with the first appearance of a non-zero Reynolds stress gradient and ending when the ordering of the terms in the mean momentum equation first becomes qualitatively representative of the high Reynolds number regime. Thus, the present effort primarily explores the mechanisms that follow the onset of nonlinear interactions. Given this, we only seek to establish a background context sufficient to further this aim.

\subsection{A scenario for the instability stage}

Numerous studies have explored the initiation and growth of the instabilities and bifurcation sequences in parallel shear flows, including the initial flow development associated with these processes. These are described in a vast number of papers and monographs, e.g. see Boiko et al. (2002) and Criminale, Jackson \& Joslin (2003) and the references therein. Indeed, the instability processes leading to the appearance and growth of nonlinear interactions between the fluctuations are often taken to effectively define transition.

For the present purposes, the instability route to the nonlinear stage described by Orszag \& Patera (1983) provides a useful context - although it is probably not as universal as they initially surmised. This scenario consists of three steps: (i) primary linear instability of the base shear flow, (ii) nonlinear saturation of the primary instability and the establishment of a secondary flow and (iii) the linear instability of the secondary flow. One attractive feature of this scenario is that it has wide applicability, in that the secondary instability phase is shown to have potential relevance to channel, boundary layer and Couette flows. Although the process they describe involves an intermediate bifurcation, unlike other routes to turbulence (such as in Taylor Couette or Rayleigh-Bernard flows) this scenario is highly compressed in a narrow Reynolds number range which they describe as 'snapthrough' transition. Note, however, that other by-pass-like mechanisms may also be operative. As described relative to figure 7 herein, this initiating process is nominally associated with the rapid rise in the skin friction after the initial instability of the base flow. Furthermore, the secondary instability of this scenario leads to a rapid threedimensionalization of the vorticity field. Once this three-dimensionalization occurs, 
and regardless of the exact details pertaining to its initiation, nonlinear mechanisms attain increasing dominance.

\subsection{Describing mean dynamics in the nonlinear stage}

Our central interest is on how, once the nonlinear mechanisms become operative, the mean dynamics develop into a structure that is physically and mathematically representative of the fully turbulent state. Toward this objective, the mathematical formulation considers incompressible, constant property, fully developed pressure driven flow between two parallel planar surfaces of large extent. The spacing between the two surfaces is $2 \delta$. The bulk flow is in the $x$-direction, and $y$ is the coordinate normal to the surfaces. The velocity components in the $x$ - and $y$-directions are given by variants of $u$ and $v$, respectively. A tilde is used to denote an instantaneous velocity component, while an upper case or an overbar is used to denote time-averaged quantities. Fluctuations about the mean are lower case.

In the case of steady laminar channel flow, the appropriately simplified form of the Navier-Stokes equation is

$$
0=-\frac{\mathrm{d} \tilde{p}}{\mathrm{~d} x}+\mu \frac{\mathrm{d}^{2} \tilde{u}}{\mathrm{~d} y^{2}},
$$

where $\mu$ is the dynamic viscosity and $\tilde{p}$ is the pressure. This differential statement of Newton's second law indicates that the dynamics of laminar flow are everywhere characterized by a balance between the retarding viscous stress gradient (viscous force) and the pressure gradient (driving normal force). In this case, each of these forces is constant across the entire channel half-width. A graphical representation of this balance is given by plotting the ratio of the viscous force to the pressure force versus position from the wall. This graph (not shown) simply consists of a horizontal line at -1 extending from $y=0$ to $y=\delta$. This, of course, reflects that the balance is everywhere accomplished via the two equal, constant and opposite forces given in (1.1).

In the transitional regime the flow is statistically stationary, and thus the Reynolds averaged Navier-Stokes (RANS) equation is employed. The appropriately simplified form of the RANS equation is given by

$$
\left.\begin{array}{l}
0=-\frac{\mathrm{d} P}{\mathrm{~d} x}+\mu \frac{\mathrm{d}^{2} U}{\mathrm{~d} y^{2}}-\rho \frac{\mathrm{d} \overline{u v}}{\mathrm{~d} y}, \\
0=A+B+C .
\end{array}\right\}
$$

Owing to correlated velocity fluctuations, the time-mean statement of dynamics now involves three quantities. Term $A$ is the mean pressure gradient that drives the flow, $B$ is the mean viscous force (viscous stress gradient) and term $C$ is the gradient of the so-called Reynolds stress. Given this naming convention, it is worth emphasizing that $C$ is in actuality the net time-averaged effect of turbulent inertia. For future reference, it is useful to present (1.2) in its inner-normalized form

$$
0=\frac{1}{\delta^{+}}+\frac{\mathrm{d}^{2} U^{+}}{\mathrm{d} y^{+2}}+\frac{\mathrm{d} T^{+}}{\mathrm{d} y^{+}},
$$

where the pressure gradient term has been evaluated using the fact that the pressure force acting on the flow cross-section is equal to the shear force acting on the upper and lower walls. In (1.3) $T=-\rho \overline{u v}$, a superscript + denotes normalization by the kinematic viscosity, $v=\mu / \rho$, and the friction velocity, $u_{\tau}=\sqrt{\tau_{w} / \rho}$, where $\tau_{w}$ is the mean wall shear stress, and $\rho$ is the mass density. Note also that $\delta^{+}=\delta u_{\tau} / v$ is the 


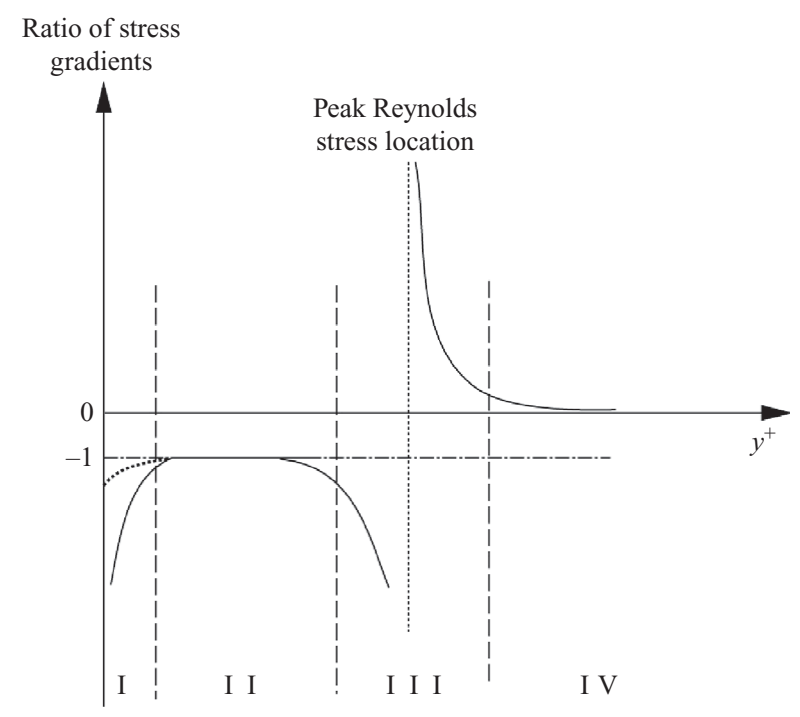

FIGURE 1. Sketch of the ratio of terms $B / C$ revealing the four force balance layers of turbulent wall-bounded flows at any fixed Reynolds number (adapted from Wei et al. 2005a). The dotted line in layer I denotes the zero pressure gradient turbulent boundary layer. This flow is different from that of channel and pipe flow in that all of the terms in the time averaged momentum equation are zero at the wall.

Reynolds number (Kármán number) based upon the channel half-height and friction velocity.

Even though the differential force balance now has three terms, one can still use the ratio of any two to expose how the balance expressed by (1.2) is realized. At any fixed Reynolds number the pressure gradient force is constant across the entire layer, and thus it is generally most instructive to examine the ratio of the other two terms, especially with increasing Reynolds number as the relative magnitude difference between the dominant and higher order terms becomes increasingly distinct. Detailed analyses that begin with this representation have been conducted for the pressure driven channel, as well as a number of other turbulent wall flows. These have revealed numerous new results of mathematical and physical significance (Fife et al. 2005a; Wei et al. 2005a,b,c; Klewicki et al. 2007; Wei, Fife \& Klewicki 2007; Metzger, Adams \& Fife 2008; Fife et al. 2009). Figure 1 displays a schematic representation of the ratio of terms $B / C$ in (1.2) at fixed Reynolds number in the fully turbulent regime. As in the laminar case, this sketch effectively reflects the mean free-body diagram for the differential fluid elements at each position within the flow. Of course, a non-trivial statement of dynamics requires that (1.2) attain a balance. This can involve either two or three codominant terms. Wei et al. (2005a) revealed that the balance of (1.2) is attained owing to a different ordering of terms in four distinct layers. Specifically, in layer I, $|A| \simeq|B| \gg|C|$; layer II, $|B| \simeq|C| \gg|A|$; layer III, $|A| \simeq|B| \simeq|C|$; layer IV, $|A| \simeq|C| \gg|B|$.

\subsection{Mean flow theory in the four-layer regime}

A number of the mathematical and Reynolds number scaling properties associated with the $U$ and $T$ profiles in the four-layer regime have been analytically determined and empirically verified (Fife et al. 2005b, 2009; Wei et al. 2005a; Klewicki et al. 2009). Central to these analyses is the finding that the equations of motion can be 


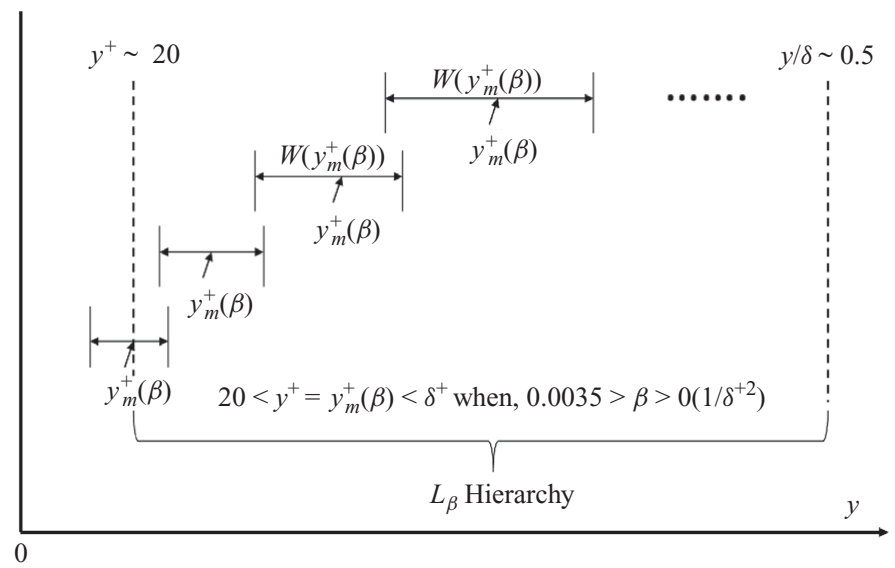

FIGURE 2. Schematic representation of the continuous hierarchy of scaling layers, the $L_{\beta}$ hierarchy. On each member of the hierarchy (1.2) admits an invariant form. The characteristic length for each layer is given by $W\left(y^{+}\right)$. Each $L_{\beta}$ layer, as specified by its position and width, is centred on the maximum of a transformed Reynolds stress function, $T_{m}^{\beta}$, that is determined by the parameter $\beta$.

written in an invariant form on each of a continuous hierarchy of scaling layers, $L_{\beta}$, and that each of these $L_{\beta}$ is analogous to layer III in figure 1. That is, across each $L_{\beta}$ the local dynamics, on average, undergo a balance breaking and exchange of forces at a scale of motion that is asymptotically proportional to the distance from the wall. A schematic depiction of the $L_{\beta}$ hierarchy structure is given in figure 2. As shown, the smallest layer on the continuous hierarchy begins deep within layer II of figure 1 and ends at a point deep within layer IV. (Layer III is an $L_{\beta}$ layer, and, in fact, is rightfully described as the average layer on the hierarchy.) Key attributes of the $L_{\beta}$ hierarchy are that each member has a well-defined characteristic length (width of the layer), and that these widths vary with distance from the wall as determined by the parameter $\beta$, see (1.7) below. As is now briefly described, all of these properties stem from the fact that (1.2) admits an invariant form on each of the $L_{\beta}$ layers, a self-similarity that can be succinctly expressed in terms of the scaled second derivative of the Reynolds stress.

The fundamental condition for dynamical self-similarity on the $L_{\beta}$ hierarchy has particular relevance to the present observations. This condition states that the quantity

$$
A(\beta)=-\frac{\mathrm{d}^{2} \hat{T}^{\beta}}{\mathrm{d} \hat{y}^{2}}(0)=-\beta^{-3 / 2} \frac{\mathrm{d}^{2} T^{+}}{\mathrm{d} y^{+2}}
$$

becomes $O(1)$ on the $L_{\beta}$ hierarchy, and over an internal subdomain may attain exact or approximate constancy (Fife et al. 2005a, 2009). In (1.4), the 'hat' denotes normalization using the characteristic length scale on each $L_{\beta}$, the superscript $\beta$ refers to the transformed Reynolds stress function associated with each value of the parameter $\beta$ (the equation for evaluating $\beta$ is given below) and the ' 0 ' refers to evaluation at the maximal value of $\hat{T}^{\beta}$ for each $L_{\beta}$. Essentially, (1.4) states that there is a region of concave downward curvature in the $T^{+}=-\overline{u v}{ }^{+}$profile for which (1.3) admits an exact self-similar form. At any finite $\delta^{+}$this dynamical self-similarity is approximate, and the level of approximation improves as $\delta^{+} \rightarrow \infty$. The existence of this self-similarity effectively constitutes a closure for (1.3) (Klewicki et al. 2009). 
Direct integrations using this closure yield the logarithmic mean velocity profile

$$
U^{+}=\frac{A^{2}}{4} \ln \left(y^{+}-C\right)+D,
$$

and in doing so reveal that logarithmic dependence is exclusively restricted to the $L_{\beta}$ hierarchy. As indicated in (1.5), the condition for dynamic self-similarity (1.4) explicitly appears in these analyses. Namely, for channel flow the leading coefficient on the logarithmic law (effectively, von Kármán's constant/coefficient) equals $A^{2} / 4$. (Note that in (1.5) $A$ is given by (1.4) and $C$ and $D$ are constants.) Analyses of data from fully turbulent channel flow verify that $A(\beta)$ only retains relevance to logarithmic dependence up to the wall-normal position of maximal length scale on the hierarchy (Klewicki et al. 2009). As made apparent by (1.4) this occurs at the point where the curvature of the Reynolds stress profile passes through zero, and thus no longer exhibits the requisite concave downward curvature. Equivalently, this also corresponds to the point where $\left|\mathrm{d} T^{+} / \mathrm{d} y^{+}\right|$is maximal.

A primary characteristic of the $L_{\beta}$ hierarchy is its associated length distribution, $W$, which measures the width of each $L_{\beta}$ as a function of $y^{+}$. Empirically verified mathematical analysis reveals that $W$ becomes an increasingly linear function of $y^{+}$as $\delta^{+} \rightarrow \infty$, e.g. Fife et al. (2005a). For turbulent channel flow this length scale distribution is given by

$$
W\left(y^{+}\right)=O\left(\beta^{-1 / 2}\right)
$$

where the values of the parameter $\beta$ can be evaluated using

$$
\beta=\frac{\mathrm{d} T^{+}}{\mathrm{d} y^{+}}+\frac{1}{\delta^{+}} .
$$

The theory specifies that through its connections to $A(\beta)$ the asymptotically linear behaviour of $W$ is directly related to the existence and value of the leading coefficient in the logarithmic equation for the mean velocity. Namely, the slope of $W, \mathrm{~d} W / \mathrm{d} y^{+}$, equals $\sqrt{\kappa}$. The scaling hierarchy upon which $W\left(y^{+}\right)$is defined has been estimated to have its smallest $L_{\beta}$ layer centred near $y^{+}=20$, while the outer edge of its largest layer ends near $y / \delta=0.5$. An estimate of $\mathrm{d} W / \mathrm{d} y^{+}$utilizing the $\delta^{+}=2003$ direct numerical simulations (DNS) data of Hoyas \& Jimenez (2006) indicates that the leading coefficient for the logarithmic equation is $0.391 \pm 0.004$ (see Klewicki et al. 2009).

\subsection{Objectives}

Primary objectives of the present effort include clarifying how the flow develops from the simple laminar channel flow force balance, expressed by the -1 ratio of terms in (1.1), to produce the ordering of terms associated with the layers of figure 1. An important element of this is to describe how the dynamical mechanisms at play during this formative regime connect to those that underlie the prevalent mean flow properties of the four-layer regime. These efforts will be aided by drawing upon the first-principles-based mean flow theory just described.

\section{Data employed}

The data used herein are derived from the DNS of Laadhari (2002) (at $\delta^{+}=72,90$ and 120), Kuroda, Kasagi \& Hirata (1989) (at $\left.\delta^{+}=100\right)$ and Hoyas \& Jimenez (2006) (at $\delta^{+}=186,547,934$ and 2003). These simulation data are supplemented with data from the physical experiments of Elsnab et al. (2010) that employed the line version of molecular tagging velocimetry (MTV). MTV is not as well known as other methods, and thus a brief summary of these experiments is now given. 
The MTV measurements were acquired in fully developed high aspect ratio microchannel water flow. Although developed in a channel having a cross-section of only $509 \mu \mathrm{m} \times 5.99 \mathrm{~mm}$, a primary conclusion of Elsnab et al. (2010) is that, over the Reynolds number range explored, these flows exhibit no discernible differences from smooth-wall macroscale channel flows. This was shown to within the uncertainty of the measurements over the laminar and transitional regimes for quantities such as the mean velocity profile, ratio of the centreline velocity to spatially averaged velocity, friction factor and other wall shear stress measures, as well as profiles of the Reynolds stress and turbulence production. Commensurate with these results, the recent particle imaging velocimetry (PIV) measurements of Natrajan \& Christensen $(2007,2009)$ in $536 \mu \mathrm{m}$ tubes also reveal flow behaviours that are essentially indistinguishable from what is seen in macroscale flows, and exhibit important features that are highly similar to those of the present measurements.

The experiments employed the MTV line method (Hill \& Klewicki 1996; Koochesfahani \& Nocera 2007). The basic aim of MTV is to track the short-time displacement of a pattern of excited long-lifetime phosphor that has been dissolved in solution (see Koochesfahani \& Nocera 2007 and references therein). For the present application $55 \mathrm{~mJ}$ pulse $\mathrm{s}^{-1} 308 \mathrm{~nm}$ excimer laser light was used to excite a single line. The phosphor employed was the ternary molecular complex developed by Ponce $e t$ al. (1993). This chemical was dissolved in water at $2 \times 10^{-4} \mathrm{M}$ concentration. Because of this low concentration, the density and viscosity remained imperceptibly different from that of pure water. Data were acquired in the form of undeformed/deformed line image pairs using an analog 8-bit gated intensified video camera, and were subsequently digitized. Descriptions of the optical set-up, camera and laser timing, as well as other experiment implementation details, are given in Elsnab (2008) and Elsnab et al. (2010).

The linear transfer function of the MTV technique requires the measurement of the line displacement, along with the time delay over which the line displaces. Each axial line displacement, $\Delta x$, was determined to within an uncertainty of \pm 0.5 pixel. Using the channel height and wall locations, a calibration constant, $c$, was determined to convert from pixel to dimensional space. The uncertainty in channel height was $4.15 \mu \mathrm{m}$. Using the method outlined by Moffat (1988), the relative uncertainty in the calibration constant was determined to be $7.64 \times 10^{-2} \mu \mathrm{m}$ pixel $^{-1}$. Axial velocities were computed using $\Delta x, c=3.10 \mu \mathrm{m}$ pixel $^{-1}$, and the measured time delay between successive images, $25 \pm 0.1 \mu$ s. The average uncertainty of the axial velocity across the channel over all Reynolds numbers investigated was less than $3.0 \%$. The primary contributor to this derives from the uncertainty associated with $c$ for each experiment. This is effectively a bias error from one experiment to the next associated with locating the exact position of the walls. This bias error primarily accounts for profile-to-profile variations. Within each profile, however, it is estimated that averaging reduced the point-to-point uncertainty to about $0.3 \%$.

Mean velocity profile measurements were acquired along the spanwise centreline at a streamwise location $x / D_{h}>290$ from the inlet of the microchannel, where $D_{h}$ is the hydraulic diameter. As described by Elsnab et al. (2010) the laminar flow profiles from these data sets were each averaged over 900 independent profile realizations, and the transitional and turbulent flow profiles were averaged over 1900 statistically independent realizations. Data reduction employed the $\mu \mathrm{MTV}$ correlation method as described in Maynes \& Webb (2002), augmented with the along-line processing technique of Thurlow \& Klewicki (2000). The MTV line technique outputs an axial velocity measurement at every pixel of the digital image. In the present experiments, this yielded a measurement every $3.10 \mu \mathrm{m}$. Under the most stringent condition, i.e. at 


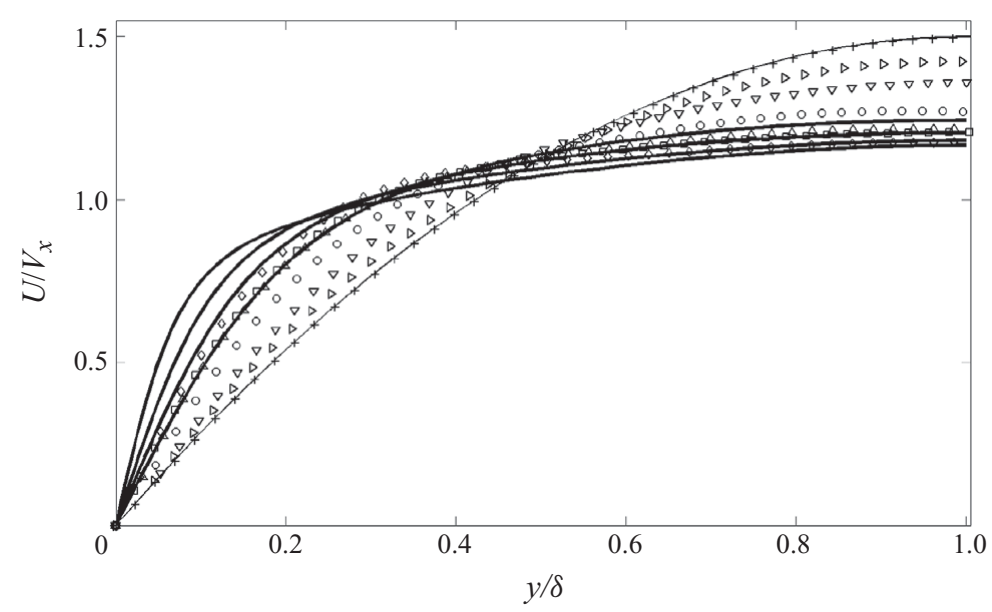

FIGURE 3. Non-dimensional mean velocity profiles from the physical experiments for $52.6 \leqslant \delta^{+} \leqslant 97.7$. The mean velocity $U$ is normalized by the spatial average (bulk) velocity $V_{x}$ and plotted versus $y / \delta$. The symbols are given in table 1 , and the fine solid line corresponds to laminar theory (Shah \& London 1978). The other lines, with progressively lower centreline values, are from the DNS studies of Laadhari $(2002)$ at $\delta^{+}=72,90$ and 120 and Hoyas \& Jimenez (2006) at $\delta^{+}=186$, respectively. For clarity, only every third measured data point is displayed in this figure.

$\begin{array}{ccccc}\delta^{+} & u_{\tau}\left(\mathrm{m} \mathrm{s}^{-1}\right) & v / u_{\tau}(\mu \mathrm{m}) & y_{m}^{+} & \text {Symbol } \\ 52.6 & 0.190 & 4.97 & \sim 17 & + \\ 54.1 & 0.197 & 4.85 & 18.1 & \triangleright \\ 57.6 & 0.209 & 4.55 & 23.6 & \nabla \\ 70.5 & 0.254 & 3.72 & 26.7 & \bigcirc \\ 81.6 & 0.292 & 3.21 & 28.3 & \triangle \\ 88.0 & 0.314 & 2.98 & 28.9 & \square \\ 97.7 & 0.345 & 2.68 & 31.3 & \diamond\end{array}$

TABLE 1. Reynolds number, friction velocity, viscous length, maximum Reynolds stress position and corresponding symbols used in figures.

the highest Reynolds number, this corresponded to a measurement every 1.16 viscous units, $v / u_{\tau}$. Light reflections associated with the present experimental configuration prevented data acquisition in the immediate vicinity of the wall, $y^{+}=y u_{\tau} / \nu \lesssim 2$. As discussed below, the experimental data were only used for $y^{+} \gtrsim 15$.

During the experiments, the temperature was continuously monitored and controlled. The pressure drop in the channel was also continuously measured in the fully developed region at the points $x / D_{h} \simeq 185$ and $x / D_{h} \simeq 350$. The friction velocity was determined from these pressure drop measurements, and was subsequently used in conjunction with $v$ to inner-normalize quantities. The uncertainty of the sensors used to obtain the pressure drop was $0.15 \%$ of full scale.

\section{Results}

\subsection{Mean velocity and Reynolds stress profiles}

The terms in the mean momentum equation (1.2) and its once-integrated form (with boundary conditions applied),

$$
u_{\tau}^{2}\left(1-\frac{y}{\delta}\right)=v \frac{\mathrm{d} U}{\mathrm{~d} y}-\overline{u v},
$$




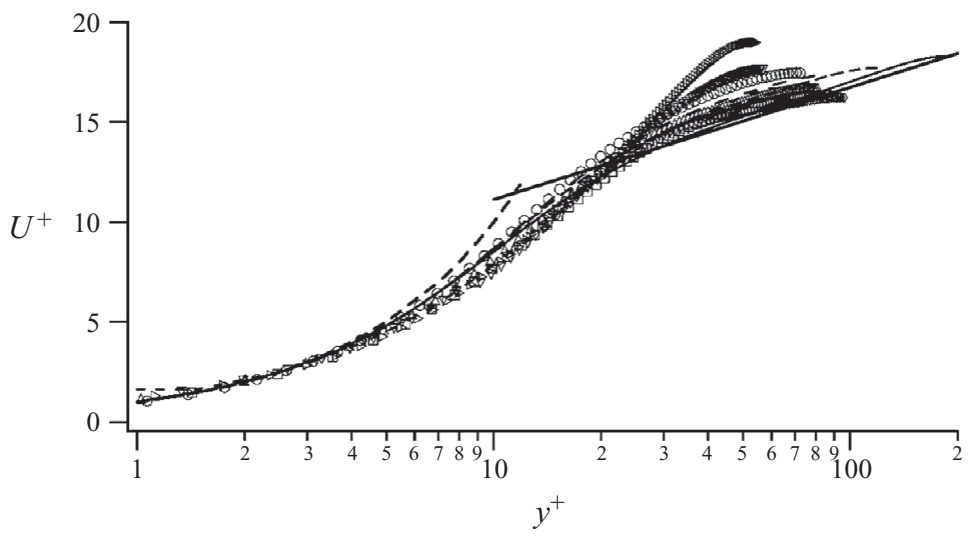

FIGURE 4. Inner-normalized mean velocity profiles for $54.1 \leqslant \delta^{+} \leqslant 97.7$ from the physical experiments, and $\delta^{+}=72,90,120$ and 186 from DNS studies. Note that in this and other figures using $y^{+}$units the $\delta^{+}$value of the profile is given by its end point. Symbols are given in table 1 , heavy dashed line, $U^{+}=y^{+}$; heavy solid line, $U^{+}=(1 / 0.41) \ln \left(y^{+}\right)+5.5$.

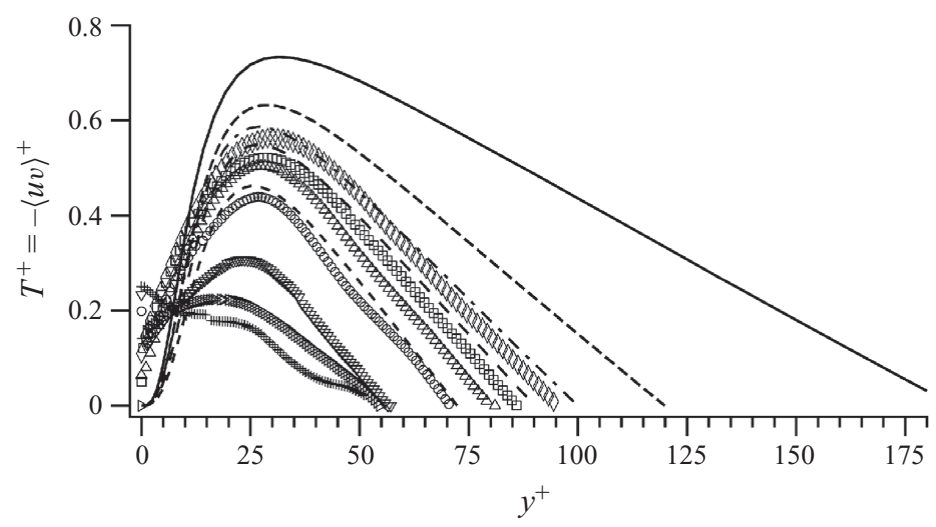

FIGURE 5. Inner-normalized Reynolds stress profiles. For the physical experiments these are obtained from the once-integrated momentum equation (3.1). The DNS data are at $\delta^{+}=$ $72,90,100,120$ and 186. Symbols are given in table 1.

may be determined using the measured mean velocity profiles and friction velocity. The indicated differentiations, however, require sufficiently smooth and closely spaced data. Owing to the attributes of the MTV technique, to the authors' knowledge the present measurements constitute some of the highest resolution mean-flow data that have been acquired in post-laminar channel flow. This is exemplified by figures 3-5, which show the mean velocity and Reynolds stress profiles.

As anticipated, figure 3 reveals that once in the non-laminar regime the mean velocity profiles continuously flatten with increasing $\delta^{+}$. The progression of the profiles depicted in figure 3 is notably similar to those presented by Orszag \& Kells (1980) for laminar channel flow subjected to perturbations consisting of a combination of finite amplitude two- and three-dimensional Orr-Sommerfeld eigenfunctions (see their figure 15). Figure 4 shows that, when plotted on the traditional semi-logarithmic axes, all of the inner-normalized mean profile measurements merge onto what appears to be a single curve for $y^{+} \lesssim 15$. These profiles, however, tend to reside slightly below 


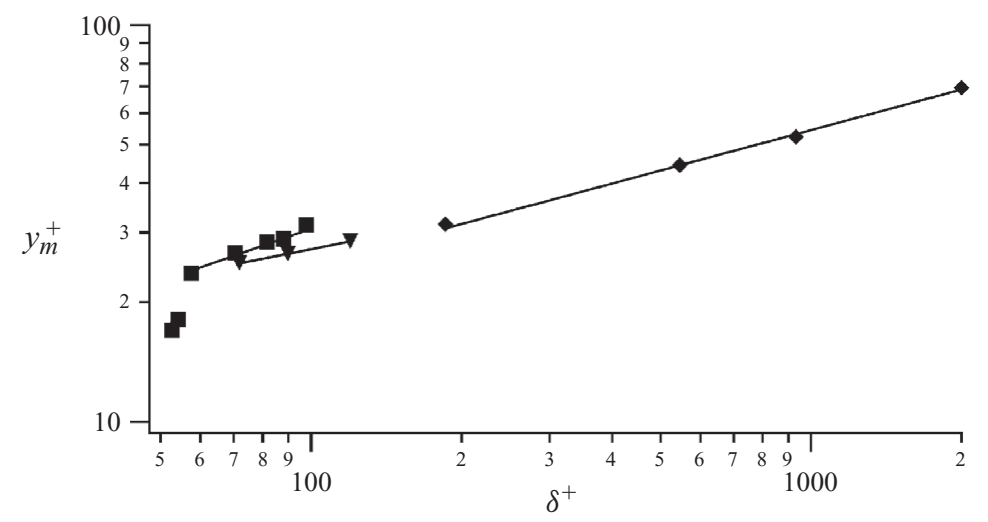

Figure 6. Position of the maximum Reynolds stress plotted versus $\delta^{+}$; $\mathbf{n}$, present experimental measurements; $\mathbf{\nabla}$, Laadhari (2002); $\bullet$, Hoyas \& Jimenez (2006).

the $U^{+}=y^{+}$line characteristic of the viscous sublayer, and similarly below the DNS data on the graph. Although almost imperceptibly small, these variations become apparent in the more sensitive measures associated with the velocity derivatives, e.g. see figure 5 as derived from (3.1). Thus, for flow behaviours in the region $y^{+} \lesssim 15$, the present analyses solely depend on the DNS results. Beyond $y^{+}=30$, the higher Reynolds number profiles in figure 4 begin to develop a logarithmic-like dependence, but to a lesser degree than exhibited by flows that are well within the four-layer regime of figure 1.

The inner-normalized Reynolds stress profiles shown in figure 5 exhibit consistent trends and develop an increasingly linear outer region profile with increasing $\delta^{+}$. For $y^{+}$positions near to and greater than the maximum, $y_{m}^{+}$, the measured profiles at $\delta^{+} \simeq 70,88$ and 98 agree quite well with the DNS profiles of Laadhari at $\delta^{+}=72$ and 90 and Kuroda et al. at $\delta^{+}=100$, although the $y_{m}^{+}$values seem to be consistently shifted by $1-3$ viscous units. The trends and profile shapes exhibited by the pipe flow PIV data of Natrajan \& Christensen (2007) (not shown) are also similar to the present channel data. Specifically, in both data sets the outer region $\left(y^{+}>y_{m}^{+}\right)$ Reynolds stress profiles $\left(T^{+}=-\overline{u v}{ }^{+}\right)$are first concave downward and then concave upward, with, of course, an inflection point between. The features associated with the curvature of the Reynolds stress profile factor significantly in how, with increasing $\delta^{+}$, the Reynolds stress gradient attains dominant order in (1.3), as well as how the terms in (1.3) evolve such that this equation admits a logarithmic mean profile solution. Owing to the near-wall sensitivities of computations using (3.1) to determine the Reynolds stress distribution, all of the experimental $\overline{u v}$ data are believed to lose accuracy for $y^{+} \lesssim 15$. This is most dramatically shown by the $\delta^{+}=52.6$ profile, whose corresponding near-wall mean profile (figure 3 ) is visually indistinguishable from the laminar profile.

For later purposes, it is useful to plot the $y_{m}^{+}$values from the profiles of figure 5 as a function of $\delta^{+}$. This is done in figure 6 . A curve-fit of the $57.6 \leqslant \delta^{+} \leqslant 97.7$ measured data indicates that the maximum Reynolds stress position, $y_{m}^{+}$values listed in table 1 , moves outward in viscous units according to $y_{m}^{+} \simeq 3.1 \delta^{+0.503}$. This is effectively the same as the $\sim \delta^{+0.5}$ rate found at much higher $\delta^{+}$, but with a larger leading coefficient. (e.g. Sreenivasan \& Sahay 1997 found that over the range $522 \leqslant \delta^{+} \leqslant 8530 y_{m}^{+} \simeq 1.8 \delta^{+0.5}$ ). Interestingly, the data of Laadhari and Hoyas and 


$\begin{array}{cccccc}\delta^{+} & \begin{array}{c}\text { Laminar } \\ (1 \text { st term })\end{array} & \begin{array}{c}\text { Turbulent } \\ (2 \text { nd term })\end{array} & C_{f_{\text {total }}} & C_{f_{\text {Dean }}} & \text { Method } \\ 52.6 & 0.0087 & 0.0016 & 0.0103 & 0.0118 & \text { Exp } \\ 54.1 & 0.0083 & 0.0028 & 0.0112 & 0.0118 & \text { Exp } \\ 57.6 & 0.0080 & 0.0037 & 0.0118 & 0.0117 & \text { Exp } \\ 70.5 & 0.0065 & 0.0049 & 0.0114 & 0.0111 & \text { Exp } \\ 81.6 & 0.0053 & 0.0052 & 0.0106 & 0.0106 & \operatorname{Exp} \\ 88.0 & 0.0050 & 0.0058 & 0.0108 & 0.0104 & \text { Exp } \\ 97.7 & 0.0044 & 0.006 & 0.0105 & 0.0101 & \text { Exp } \\ 72 & 0.0059 & 0.0042 & 0.0101 & 0.0109 & \text { DNS } \\ 90 & 0.0046 & 0.0050 & 0.0096 & 0.0102 & \text { DNS } \\ 100 & 0.0043 & 0.0055 & 0.0098 & 0.0100 & \text { DNS } \\ 120 & 0.0033 & 0.0056 & 0.0089 & 0.0094 & \text { DNS } \\ 186 & 0.0021 & 0.0061 & 0.0082 & 0.0083 & \text { DNS }\end{array}$

TABle 2. Contributions to (3.2) for each $\delta^{+}$presented in table 1 and for the DNS data at $\delta^{+}=72,90,100,120$ and 186 .

Jimenez yield curve-fits of $y_{m}^{+} \simeq 8.6 \delta^{+0.250}$ and $y_{m}^{+} \simeq 5.5 \delta^{+0.331}$, respectively, indicating that early in the nonlinear development stage there is a $\delta^{+}$range over which $y_{m}^{+}$ migrates outward more slowly than $\sqrt{\delta^{+}}$. Further evidence of this is given by the fact that the two higher Reynolds number $y_{m}^{+}$data points of Hoyas and Jimenez increase at a rate closer to $\delta^{+0.5}$ than their two lower $\delta^{+}$data points.

\subsection{Skin friction evolution}

Mean momentum field development is accompanied by associated changes in the surface shear stress. As such, the behaviours of the skin friction with increasing $\delta^{+}$ are also useful for more precisely specifying what is described herein as the transitional regime.

As discussed by Fukagata, Iwamoto \& Kasagi (2002), the local skin friction, $C_{f}$, can be decomposed into laminar and turbulent contributions according to

$$
C_{f}=\frac{12}{R e_{b}}+12 \int_{0}^{1}(1-\eta)(-\overline{u v}) \mathrm{d} \eta,
$$

where $R e_{b}=2 \delta V_{x} / v, V_{x}$ is the bulk velocity, i.e. the average of the mean profile across the channel, $\eta=y / \delta$ and $\overline{u v}$ is normalized by $4 V_{x}^{2}$. Fukagata et al. describe the first term on the right of (3.2) as the laminar contribution and the second term as the turbulent contribution. Note that the latter consists of a weighted integral of the Reynolds stress, and thus with increasing $\delta^{+}$the laminar contribution becomes a decreasing fraction of the total. The laminar and turbulent contributions to the skin friction were computed using (3.2), and are presented in table 2. This table also includes the $C_{f}$ values found using Dean's correlation (Dean 1978). The estimates based on (3.2) are generally in good agreement with Dean's correlation, although it should be noted that Dean's correlation is intended for use at higher Reynolds numbers. Examination of the measurements also reveals that the estimates of $C_{f}$ determined from the mean velocity gradient at the wall are consistently about $20 \%$ lower than both Dean's correlation and (3.2) (see Elsnab et al. 2010). As shown later in figure 15, this is because the present mean gradient profiles lose accuracy in the region $y^{+} \lesssim 15$. This loss of accuracy near the wall also influences the integrations 


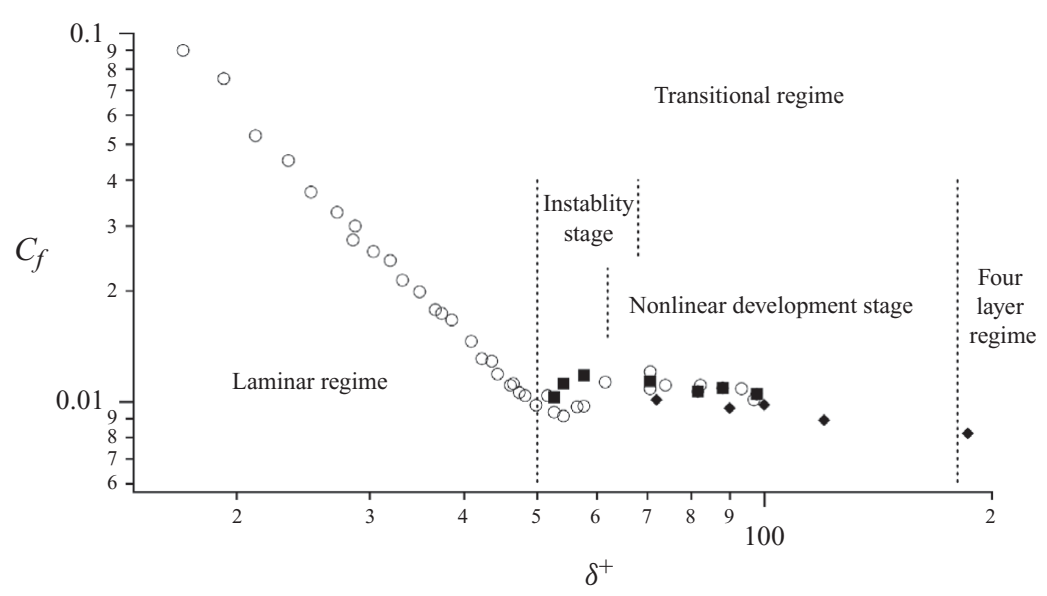

FIGURE 7. Skin friction coefficient plotted versus $\delta^{+}$; O, data of Elsnab et al. (2010) based upon pressure drop measurements; $\mathbf{\square}$, present experimental measurements derived from (3.2); $\downarrow$, DNS data of Kuroda et al. (1989), Laadhari (2002) and Hoyas \& Jimenez (2006).

of (3.2) owing to the fact that the Reynolds stresses were computed from (3.1). The net effect is that the computed contributions from the Reynolds stress erroneously occur closer to the wall. This occurs owing to higher experimental Reynolds stress values for $y^{+} \lesssim 15$ and the linear weighting factor in (3.2) that decreases with distance from the wall. For these reasons, the integrations of the measured profiles utilize a linear approximation for the Reynolds stress between the wall and $y^{+} \simeq 10$. The results listed in table 2 indicate that at $\delta^{+}=52.6$ the laminar contribution accounts for greater than $80 \%$ of the total, while at $\delta^{+}=186$ the laminar part only accounts for about $25 \%$.

Pressure drop measurements are arguably the most accurate way to estimate the wall shear stress. Elsnab et al. (2010) used this method across the laminar, transitional and turbulent regimes and presented the data in the traditional form of the friction factor versus bulk Reynolds number (see their figure 5). Comparisons revealed very good agreement with the analytical prediction for laminar flow, as well as with established correlations for turbulent flows. Figure 7 presents these same data as $C_{f}=\tau_{w} /\left(0.5 \rho V_{x}^{2}\right)$ versus $\delta^{+}$. As is apparent, the pressure-drop-based data for $\delta^{+} \lesssim 50$ closely follow the straight line indicative of laminar flow, and then for subsequent increases in Reynolds number exhibit the expected rise marking the advent of transition. Consistent with previous observations and analyses, transition begins at $\delta^{+} \simeq 50$, or equivalently $R_{c}=\delta^{+} U_{c}^{+} \simeq 1000$ (Orszag \& Kells 1980; Orszag \& Patera 1983). The $C_{f}$ data found using (3.2) listed in table 2 are also plotted in figure 7. The pressure-drop- and (3.2)-based data are generally in good agreement. At the lowest $\delta^{+}$values in the transitional regime, the (3.2)-based data are, however, consistently higher. Comparisons with the DNS $\overline{u v}{ }^{+}$profiles indicate that this discrepancy is associated with the aforementioned over-estimation of $\overline{u v}^{+}$near the wall.

Most likely, transition begins at a Reynolds number slightly less than that corresponding to where the friction factor starts to deviate from the laminar line, to account for the initial growth of infinitesimal disturbances. The flow field then evolves in such a way that the mean effect of the unsteady dynamics leads to the abrupt rise in the contributions to $C_{f}$ resulting from the second term in (3.2). As noted at 
the outset, this abrupt rise is nominally associated with the instability stage of the transitional process. The boundary between the instability and nonlinear development stages is, however, not expected to be distinct. This is because at these low Reynolds numbers linear neutral modes are expected to continue to be excited even though some nonlinear interactions have commenced.

The mean dynamical mechanisms responsible for the rise in the turbulent contributions to $C_{f}$ are physically and mathematically represented by the terms in (1.3). The ordering of terms in (1.3) represented in figure 1 has been both empirically and analytically established for the fully turbulent state (Wei et al. 2005a). Furthermore, it has been analytically shown that this ordering is necessary for the equations to accurately admit the predominant features associated with the fully turbulent state, e.g. a logarithmic mean velocity profile. Thus, in the present context the transitional regime is deemed to end when the ordering characteristic of figure 1 is first established. According to criteria to be discussed further in $\S 3.4$, this occurs in channel flow at $\delta^{+} \simeq 180$. In the absence of any additional forces, this ordering of terms becomes increasingly well established for all subsequent increases in $\delta^{+}$. Thus, one is assured that the scalings associated with this ordering will hold to an increasingly accurate approximation as well.

\subsection{Mean momentum balances}

The evolution of the mean momentum balance is explored using graphs of the ratios of the terms in (1.3) as discussed in $\S 1$. Expressing the balances using ratios is instructive since they convey the relative magnitudes of the individual contributions.

Figure 8 shows the profiles of $\left(\mathrm{d} T^{+} / \mathrm{d} y^{+}\right) \delta^{+}(C / A$ in (1.2) or equivalently (1.3)) and $\left(\mathrm{d}^{2} U^{+} / \mathrm{d} y^{+2}\right) \delta^{+}(B / A$ in (1.2) or equivalently (1.3)). This figure naturally exhibits a number of symmetric behaviours, since, at any given Reynolds number, the pressure gradient term is constant. Any change in the ratio $B / A$ is thus accompanied by an equal magnitude change in $C / A$. In interpreting figure 8 it is also helpful to recall that the laminar flow balance is expressed by two horizontal lines at $C / A=0$ and $B / A=-1$. Dynamical evolution is therefore represented by the symmetric deformation of these two straight lines. (The near-wall profiles of figure 9 provide a clear sense of this continuous deformation.) From figure 8 it is apparent that significant changes occur from the laminar profile to the lowest Reynolds number transitional profile. The zero-crossings for $\left(\mathrm{d} T^{+} / \mathrm{d} y^{+}\right) \delta^{+}$are the maximum $T^{+}$positions, $y_{m}^{+}$, plotted in figure 6 and listed in table 1. As discussed in detail by Klewicki et al. (2007), this zero-crossing is dynamically significant, since for $y^{+}<y_{m}^{+}$the Reynolds stress gradient force behaves like a momentum source, and for $y^{+}>y_{m}^{+}$it behaves like a momentum sink. Symmetry demands that the zero-crossings of $\left(\mathrm{d} T^{+} / \mathrm{d} y^{+}\right) \delta^{+}$ also correspond to the $\left(\mathrm{d}^{2} U^{+} / \mathrm{d} y^{+2}\right) \delta^{+}$crossings of the line at -1 .

As mentioned in $\S 2$, in the immediate vicinity of the wall the measurements of Elsnab et al. (2010) are not believed to be of an accuracy sufficient to reliably reveal the flow development associated with layer I and the lower part of layer II of figure 1 . The DNS profiles shown in figure 9 are thus used to discern these near-wall behaviours. Characteristic features of the $C / A$ profiles include the aforementioned zero-crossings at increasing $y^{+}$values for increasing $\delta^{+}$(also see figure 10), the increasing positive peak in the region $5 \leqslant y^{+} \leqslant 10$ and the approach to zero as $y^{+} \rightarrow 0$. Similarly, the $B / A$ profiles exhibit a negative peak that mirrors that of the corresponding $C / A$ positive peak, and asymptote to -1 as $y^{+} \rightarrow 0$. This asymptote to -1 simply indicates that the mean pressure gradient and mean viscous force are equal at the wall, and thus automatically coincide with the Reynolds stress gradient 


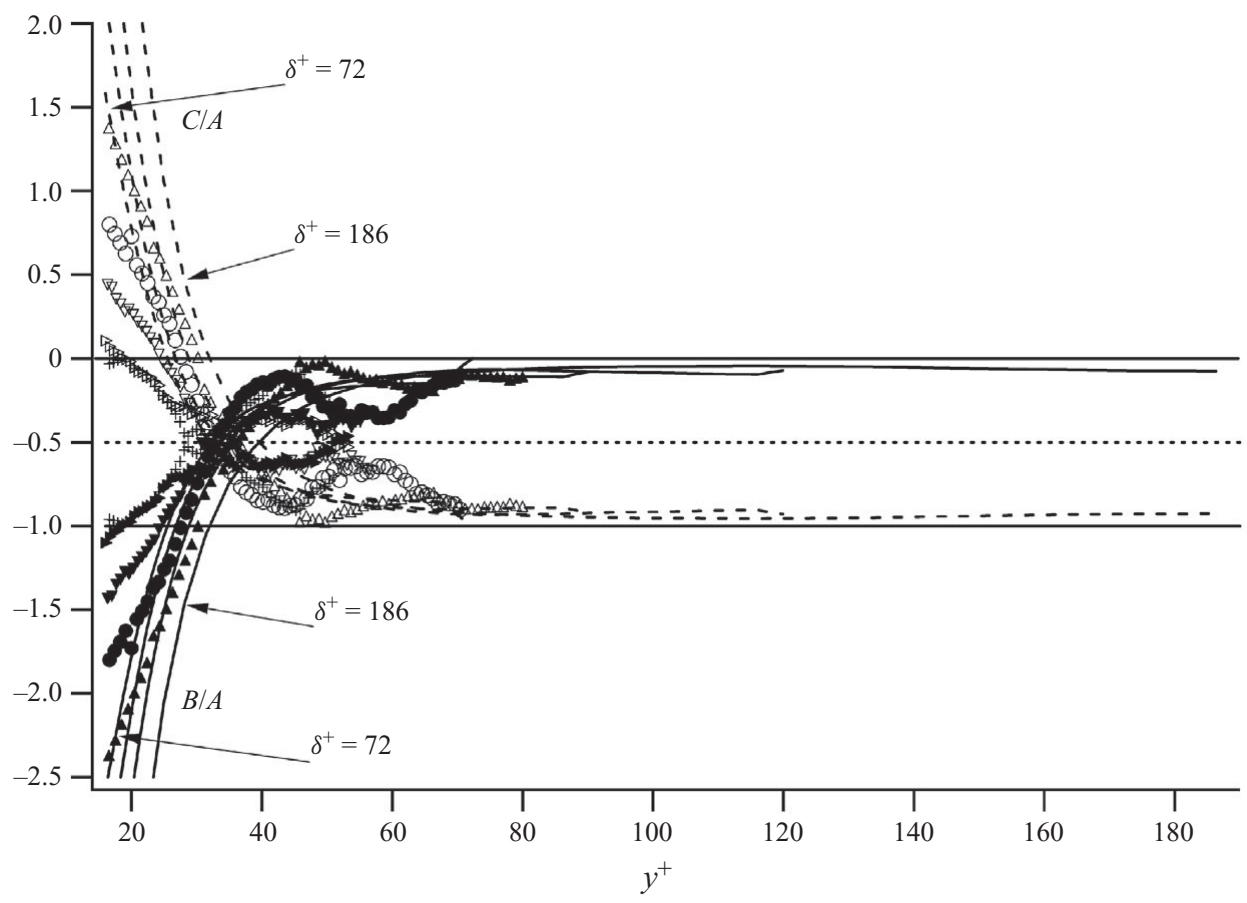

FIgURE 8. Ratio of the Reynolds stress gradient to the pressure gradient $(C / A$ in $(1.3))$ and the viscous stress gradient to the pressure gradient $(B / A$ in (1.3)). Symbol shapes are given in table 1. Filled symbols, $\left(\mathrm{d}^{2} U^{+} / \mathrm{d} y^{+2}\right) \delta^{+}$; open symbols, $\left(\mathrm{d} T^{+} / \mathrm{d} y^{+}\right) \delta^{+} ; C / A$ and $B / A$ profiles from the DNS data sets are given by dashed lines and solid lines, respectively; laminar flow $\left(\mathrm{d}^{2} U^{+} / \mathrm{d} y^{+2}\right) \delta^{+}$is given by the horizontal line at -1 . Profiles from the DNS of Laadhari (2002) are at $\delta^{+}=72,90,120$, while that from Hoyas \& Jimenez (2006) is at $\delta^{+}=186$.

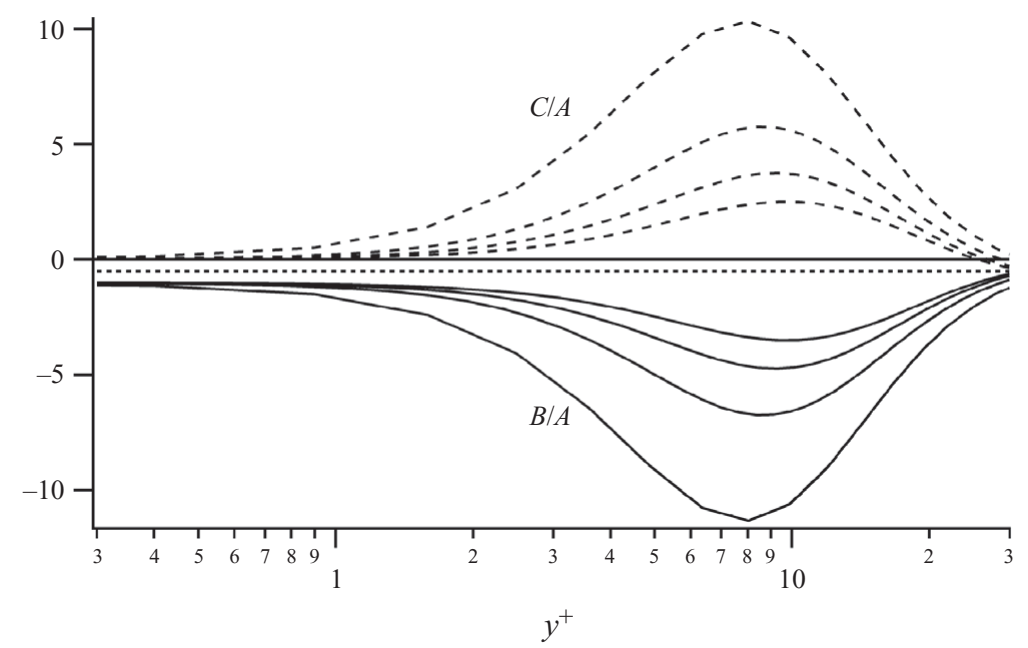

FIGURE 9. Near-wall profiles of $B / A$ (solid lines) and $C / A$ (dashed lines) from (1.3). Ascending magnitude curves are the DNS profiles at $\delta^{+}=72,90,120$ and 186, respectively. Dotted line is at -0.5 .

approaching zero in layer I. The region over which the $|B / A|$ and $|C / A|$ profiles are both much greater than unity reflects the emergence of the stress gradient balance layer (layer II) - a condition that clearly extends over a greater portion of the 


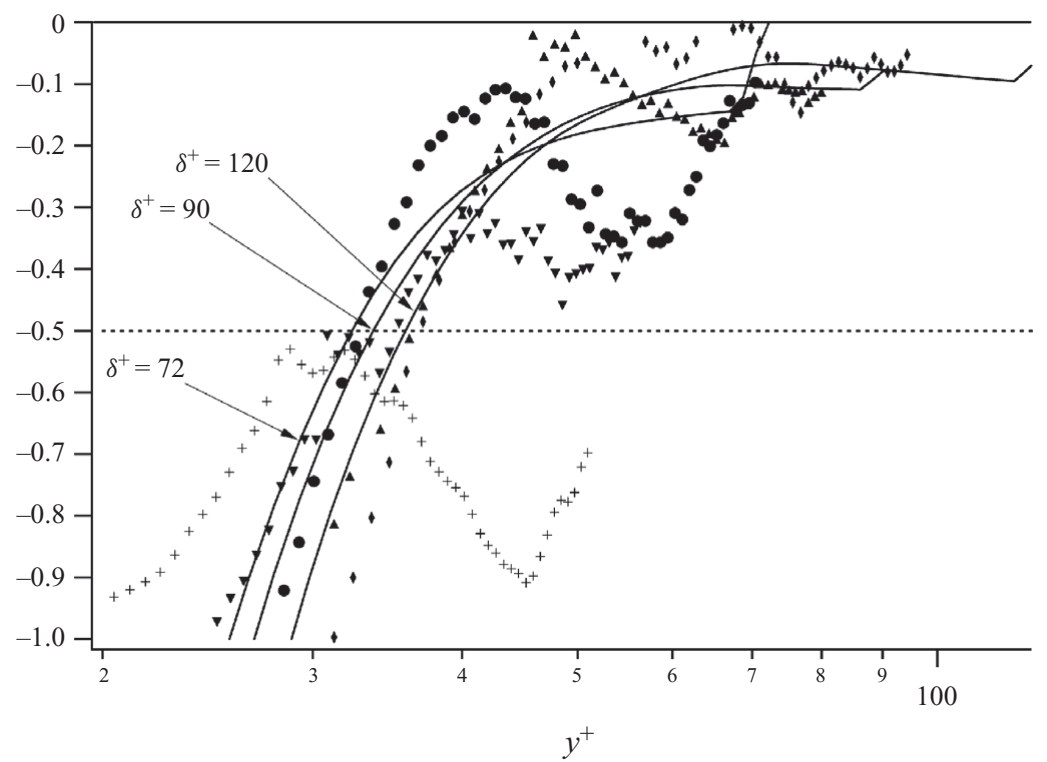

Figure 10. Expanded view of the ratio $B / A$ from (1.3). Symbol shapes are given in table 1 . Solid lines are the DNS profiles at $\delta^{+}=72,90$ and 120 .

near-wall flow as $\delta^{+}$increases. It is also apparent that with increasing $\delta^{+}$the peaks in these profiles march inward in viscous units, and thus concentrate nearer to the wall at a rate faster than the decrease in $v / u_{\tau}$. Higher Reynolds number data reveal that this peak settles into an essentially fixed position near $y^{+}=7$ (Wei et al. 2005a). The theory of $\S 1.3$ shows that the decay rate of the Reynolds stress gradient for $y^{+}$values beyond the peak determines the characteristic length scale distribution that, among other features, underlies the emergence of a logarithmic mean velocity profile as $\delta^{+}$ becomes large. Comparison with previous analyses indicates that the decay rate of the $\delta^{+}=186 \mathrm{~d} T^{+} / \mathrm{d} y^{+}$profile of figure 9 is quantitatively similar to that observed at higher $\delta^{+}$(Wei et al. 2005a).

The profiles in figure 8 exhibit complex behaviours in the region $20 \lesssim y^{+} \lesssim 100$. Detailed features of the $B / A$ profiles are more clearly exposed in the expanded view of figure 10. The profile for $\delta^{+}=52.6$ is distinctive in that it does not quite cross the -0.5 line, but rather rises up very near to this value and then returns toward -1 . This indicates the existence of an internal zone in which the mean effect of turbulent inertia $\left(\mathrm{d} T^{+} / \mathrm{d} y^{+}\right)$locally attains a value that is of the same order of magnitude as the mean pressure gradient and viscous force. For higher $\delta^{+}$all of the profiles cross -0.5 , and exhibit a characteristic shape that is exemplified by the $\delta^{+}=70.5$ data shown separately in figure 11. Behaviours exhibiting Reynolds number dependence revealed in figure 10 include the $y^{+}$position where the Reynolds stress gradient and viscous stress gradients cross (across the -0.5 line), as well as the properties of an inertial zone that continually develops over an interior range of $y^{+}$values outward of the profile crossings. This region of maximal $\left|\mathrm{d} T^{+} / \mathrm{d} y^{+}\right|$is necessarily centred on the $y^{+}>y_{m}^{+}$inflection point across which the $-\overline{u v}$ profile changes from concave downward to concave upward curvature. As indicated by the progression of the profiles at $\delta^{+}=70.5,81.6,90,97.7$ and 120 in figure 10, the local maxima increase in magnitude and monotonically move outward in $y^{+}$units with increasing $\delta^{+}$. 


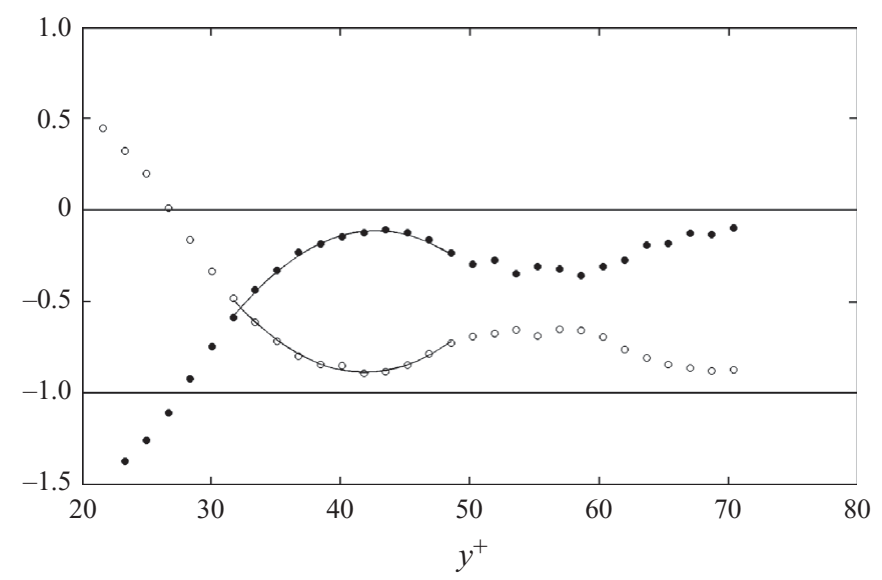

FIgURE 11. The profiles of $B / A$ (filled symbols) and $C / A$ (open symbols) from figure 8 at $\delta^{+}=70.5$. This figure illustrates the characteristic shape of the measured profiles.

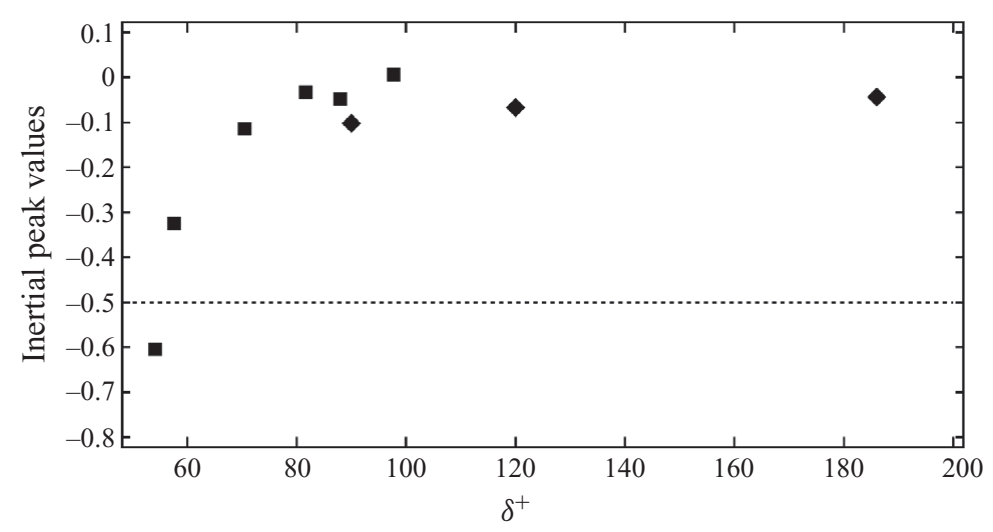

FIGURE 12. Normalized local minimum viscous stress gradient values $(B / A)$ associated with the inertial zone centre positions given in figure $13(a)$, $\mathbf{\square}$, experimental measurements; $\bullet$, DNS data.

The profiles of Elsnab et al. (2010), as well as those by Natrajan \& Christensen (2007) for pipe flow (not shown), exhibit a much more noticeable change in curvature than the DNS profiles. The DNS data do, however, provide evidence for this internal inertial zone, but only less distinctly and not for the $\delta^{+}=72$ profile. Significantly, all available DNS channel data at higher $\delta^{+}$also exhibit this change in curvature. This property has been analytically shown to be required for (1.3) to admit (either exactly or approximately) a logarithmic mean profile (Fife et al. 2009; Klewicki et al. 2009). The reasons for the quantitative difference between existing measurements and the DNS results are not entirely clear. In $\S 4$ we speculate that these observed differences stem from differences in the initial distribution of linearly unstable modes.

Local curve-fits of the type depicted in figure 11 allow the peak amplitude and position of the inertial zone to be quantified. The peak values associated with the inertial zone are plotted versus $\delta^{+}$in figure 12. At the lowest Reynolds number shown, the viscous and Reynolds stress gradients nominally combine to offset the pressure gradient force within this emerging inertial zone. With increasing Reynolds number, however, it is clear that the mean force balance within this zone continually progresses 
(a)

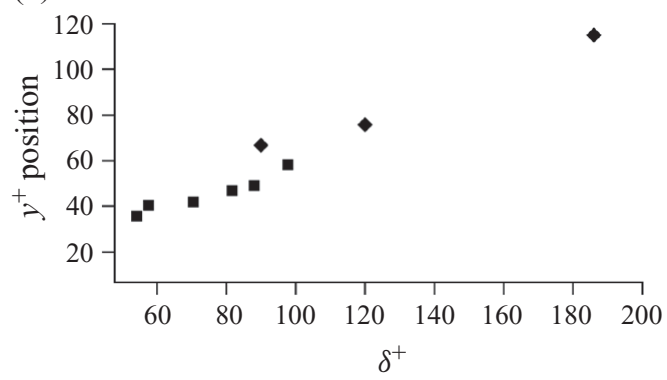

(b)

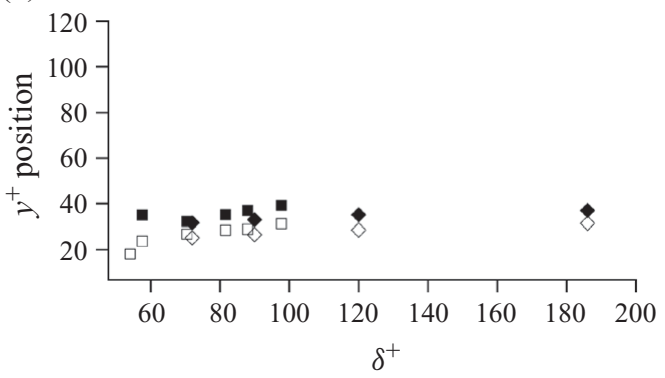

FIgURE 13. (a) Centre position of the interior inertial zone (see figure 11) plotted as a function of $\delta^{+}$: physical experiments; $\mathbf{\square}$, DNS; $\bullet$. $(b)$ Positions where the viscous and Reynolds stress gradient profiles cross as a function of $\delta^{+}$: physical experiments; $\mathbf{\square}$ DNS; $\bullet$ Open symbols are corresponding $y_{m}^{+}$values.

toward one that is dominated by the pressure gradient and Reynolds stress gradient. (Recall that the values plotted in figure 12 are normalized by the pressure gradient.) At $\delta^{+}=186$, it is estimated that at the inertial peak the viscous force accounts for about $5 \%$ of the balance. Figure $13(a)$ shows the estimated peak positions (minimum in $\mathrm{d} T^{+} / \mathrm{d} y^{+}$, inflection in $T^{+}$) for varying $\delta^{+}$. These positions progressively move outward in $y^{+}$units. They also meld (albeit not perfectly) with the DNS data trend for $\delta^{+}>90-$ even though the peaks are more distinct in the profiles from the physical experiments. As with the peak positions, the peak values from the DNS profiles (figure 12) also exhibit a consistent trend and nominally meld with the measurements.

Figures 8 and 10 contain attributes having recognizable association with layer III of figure 1. Namely, one can discern that the Reynolds stress gradient and viscous stress gradient profiles cross at a point near the wall but beyond $y_{m}^{+}$. This crossing occurs at all but the lowest Reynolds number represented in figure 10. It results because of the inertial zone formation just discussed, and also occurs at increasing $y^{+}$with increasing $\delta^{+}$. Owing to symmetry, these profiles always cross when each has a value of -0.5 . At the crossing point, each stress gradient has a magnitude that is $50 \%$ of the pressure gradient (term $A$ ). Relative to the four-layer regime momentum balance, the position where these profiles cross consistently lies just interior to the outer boundary of layer III. Wei et al. (2005a) subjectively, but self-consistently, define the outer boundary of layer III as the position where the ratio of terms $B / C$ in (1.2) equals 0.5 . As constrained by (1.2) this corresponds to the ratio of terms $B / A$ and $C / A$ being -0.33 and -0.67 , respectively. These observations give reason to expect that the positions of the crossings scale with the characteristic length associated with the position of $y_{m}$. In the four-layer regime, $y_{m}$ (and the widths of layers II and III) scales with $\sqrt{\delta v / u_{\tau}}$, or equivalently, under inner-normalization $y_{m}^{+} \sim \sqrt{\delta^{+}}$(Wei et al. 2005a; Klewicki 2010).

The positions of the profile crossings are plotted in figure 13(b). For comparison, the $y_{m}^{+}$positions from figure 6 are also included. The crossing positions of the measured data increase more rapidly with $\delta^{+}$than those associated with the DNS data. This is self-consistent, as evidenced by the fact that the crossing positions, for either the measured or DNS data, exhibit essentially the same $\delta^{+}$dependence as do the respective $y_{m}^{+}$values. The present measurements provide evidence that soon after the appearance of a non-zero $\overline{u v}{ }^{+}\left(y^{+}\right)$profile the Reynolds number dependence of $y_{m}^{+}$ is close to $\sqrt{\delta^{+}}$. At slightly higher $\delta^{+}$, the DNS data suggest that this is followed by a 


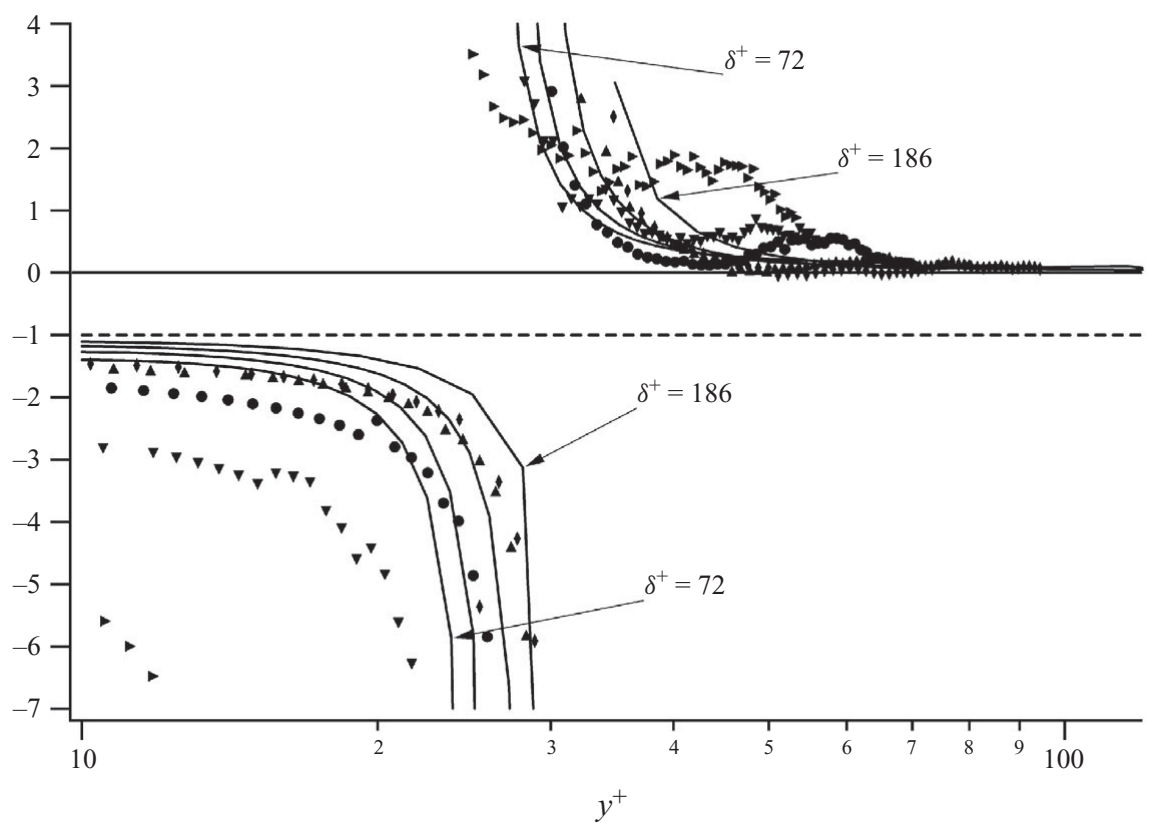

FIGURE 14. Ratio of the gradient of the viscous stress to the gradient of the Reynolds stress, $\left(\mathrm{d}^{2} U^{+} / \mathrm{d} y^{+2}\right) /\left(\mathrm{d} T^{+} / \mathrm{d} y^{+}\right)$. Symbol shapes for the data from the physical experiments are given in table 1 . DNS profiles are at $\delta^{+}=72,90,120$ and 186 .

range of $\delta^{+}$over which the rate of outward migration is less than $\sqrt{\delta^{+}}$. The higher $\delta^{+}$ DNS data, as well as other experimental data at much higher $\delta^{+}$(not shown), indicate that a dependence close to $\sqrt{\delta^{+}}$is firmly re-established as $\delta^{+}$continues to increase. Comparison of figures $13(a)$ and $(b)$ reveals that the position of the $y^{+}>y_{m}^{+}$inflection in $-\overline{u v}{ }^{+}$moves to larger $y^{+}$values more rapidly with $\delta^{+}$than does the position where terms $B$ and $C$ cross. As demonstrated in $\S 3.5$, the expanding region between these points correlates with the first appearance of an approximately logarithmic mean velocity profile. The increasing width of this inertially dominated flow eventually emerges as the inner portion of layer IV in figure 1.

Overall, the primary attributes of layer III are thus seen to effectively come into being at the $\delta^{+}$when terms $B$ and $C$ first cross. That is, this attribute ensures the existence of an interior zone in which all three terms in (1.3) are of dominant order. Furthermore, with increasing $\delta^{+}$the balance breaking and exchange of forces characteristic of layer III becomes increasingly distinct.

Figure 14 displays mean momentum balances for $y^{+}>10$ in the manner represented in figure 1 . These data clearly reveal that the profiles in the region emerging as layer II begin with large negative values at low $\delta^{+}$, and with increasing $\delta^{+}$continually progress toward a plateau at -1 . This quantifies the emergence of the Reynolds stress gradient as an important contributor to the balance expressed by (1.3) in the region interior to $y_{m}^{+}$, and its eventual co-dominance with the viscous stress gradient in this region of the flow. As required, across $y_{m}^{+}$the profiles in figure 14 are singular, heading to negative $\infty$ when $y_{m}^{+}$is approached from below and emerging from positive $\infty$ on the other side of $y_{m}^{+}$. At large $\delta^{+}$the ratio $B / C$ monotonically approaches zero for $y^{+}>y_{m}^{+}$. As described by Fife et al. (2005b), this signals the completion of the balance breaking and exchange process in which the layer II balance between the Reynolds 
stress gradient and viscous stress gradient develops into the layer III balance in which all the terms are important, and subsequently, to the one in which the Reynolds stress gradient and pressure gradient comprise the dominant order balance in layer IV. As discussed in $\S 1.3$, this balance breaking and exchange structure is central to mathematically and physically describing the mean flow properties of the four-layer regime.

During the process by which the ordering of terms underlying figure 1 is established, the ratio $B / C$ does not monotonically approach zero for $y^{+}>y_{m}^{+}$. The profiles of figure 14 for $y^{+}>y_{m}^{+}$develop toward the inertially dominated condition characteristic of layer IV at large $\delta^{+}$through the simultaneous appearance of one inertial zone that starts out being near $y^{+}=y_{m}^{+}$, and to a less dramatic extent, another near $y^{+}=\delta^{+}$. The inertial zone nearer to $y^{+}=y_{m}^{+}$is associated with the inflection point in the $-\overline{u v}{ }^{+}$profile, while, as suggested below, the zone nearer to $y^{+}=\delta^{+}$appears to be a natural consequence of the wallward concentration of mean vorticity. Thus, during the nonlinear development stage there is a portion of the flow sandwiched between these two inertial zones in which the viscous force retains elevated importance. This phenomenon is perhaps most clearly exemplified in the $\delta^{+}=70.5$ profile of figure 11 , but is also evident, albeit more subtly, in the other experimental and DNS profiles (see figure 10). Specifically, the inertial region that is associated with the inflection in $-\overline{u v}^{+}$, whose location is quantified in figure $13(a)$, resides interior to a localized residual layer of elevated, yet diminishing, viscous force.

The behaviours of the $B / C$ ratio for $y^{+} \lesssim 10$ can be deduced from figure 9 . For $y^{+} \gtrsim 3$ the balance expressed by (1.3) is increasingly well approximated by $B$ equaling $-C$, and for $y^{+} \rightarrow 0, C \rightarrow 0$ and $B \rightarrow-A$.

\subsection{Establishment of the four-layer ordering}

The data of figure 14 demonstrate that through the nonlinear development stage (as defined relative to figure 7) the operative force balance smoothly evolves toward the structure of figure 1. Pertinent to this, there is now a cogent, first-principles-based mathematical theory that leverages the underlying ordering of terms. By doing so, this theory informs us how and why the predominant characteristics of the four-layer regime exist (Fife et al. 2005b, 2009; Klewicki et al. 2009). In this subsection and the next, the focus is on the connections between the dynamics in the transitional and four-layer regimes. We begin by estimating the minimum Reynolds number at which the high Reynolds number ordering is first established. As noted in $\S 3.2$, this will also serve to delineate the upper end of the transitional regime.

Klewicki et al. (2007) (also see Klewicki 2010) describe the characteristic properties of the four-layer structure. These include the ordering of terms described relative to figure 1, as well as the scaling behaviours associated with the widths of these layers and velocity increments across them. Two criteria relating to the ordering of terms in layers II and IV are employed, and then supplemented by verifying the layer scaling properties. In layer II the requisite ordering is given by $|B| \simeq|C| \gg|A|$, while in layer IV it is given by $|C| \simeq|A| \gg|B|$. In each case, these orderings are (subjectively) said to be established when the smaller of the two dominant terms is ten times larger than the smallest term (e.g. when $|C / A|>10$ somewhere in layer II). Examination of the $\delta^{+}=120$ data reveals that $|C / A|$ attains a maximal value of about 5.8 in layer II, while in layer IV $|A / B|_{\max } \simeq 14$.9. Thus, at this $\delta^{+}$only one of the two criteria is satisfied. From this, it is also seen that layer IV settles into its high Reynolds number ordering more rapidly than layer II. At $\delta^{+}=186$ the layer II criterion is just barely met $\left(|C / A|_{\max } \simeq 10.2\right)$, while $|A / B|_{\max } \simeq 22.1$ in layer IV. Thus, it is estimated that 


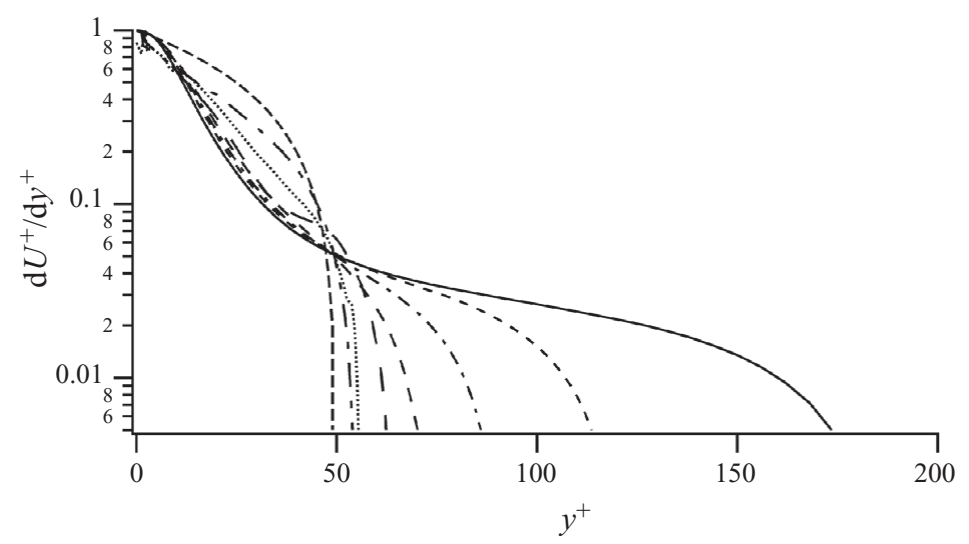

FIGURE 15. Inner-normalized profiles of the mean velocity gradient; - - -, laminar profile $\left(\delta^{+}=50\right),--_{-}, \delta^{+}=54.1, \ldots \ldots ., \delta^{+}=57.6,-{ }_{-}, \delta^{+}=70.5,-\longrightarrow, \delta^{+}=72,--\longrightarrow, \delta^{+}=90$, $--, \delta^{+}=120,-, \delta^{+}=186$.

the four-layer structure is first established at about $\delta^{+}=180$, as depicted in figure 7 . This estimate is further supported by the fact that the layer widths and velocity increments for the $\delta^{+}=180$ DNS of Moser, Kim \& Mansour (1999) adhere to the scaling properties exhibited by much higher $\delta^{+}$data, as well as derived from analyses of (1.3) that assume the ordering of figure 1 (Wei et al. 2005a).

\subsection{Mean velocity profile development}

Although the set of dynamically active mechanisms given by the terms in (1.3) is the same as in the four-layer regime, the above analysis clarifies that during the nonlinear development stage all of these mechanisms are generally non-negligible across the entire half-channel. It is, however, an unambiguous certainty that the action of these dynamical mechanisms ultimately produces the high Reynolds number ordering, and, once it is formed, the same set of mechanisms also sustains this ordering to ever-increasing magnitude disparity as $\delta^{+} \rightarrow \infty$. Such observations naturally motivate deeper inquiry into how the transitional flow dynamics connect to those responsible for the observed distribution of mean momentum at high Reynolds number.

Figure 4 reveals that the inner-normalized mean profiles begin to develop logarithmic-like tendencies with increasing $\delta^{+}$. Of course, the approach toward logarithmic behaviour arises owing to the emergence of the mean effects of turbulent inertia as represented by term $C$ in (1.3). A primary consequence of this emerging effect is the redistribution of vorticity such that a greater fraction of the total is concentrated closer to the surface. This feature of flow field evolution through both stages of the transitional regime is illustrated in figure 15 in which representative mean velocity gradient profiles from the experimental measurements and DNS are plotted. (Note that in this and subsequent plots only a subset of the measured profiles are presented so that $\delta^{+}$trends are easily discerned. Also recall that the mean vorticity is given by $\Omega_{z}=-\mathrm{d} U / \mathrm{d} y$.) For reference, this figure also shows the linear laminar profile at $\delta^{+}=50$. The logarithmic $y$-axis allows one to better see the concentration of mean vorticity toward the surface. These data reveal that in the near-wall region the rate at which vorticity concentrates wallward is at first more rapid than the rate at which the viscous length scale decreases. Note further that as the four-layer structure develops, the dynamics produce and sustain an extended region away from the wall of decreasing low-level vorticity. Classical notions prescribe that for sufficiently high 


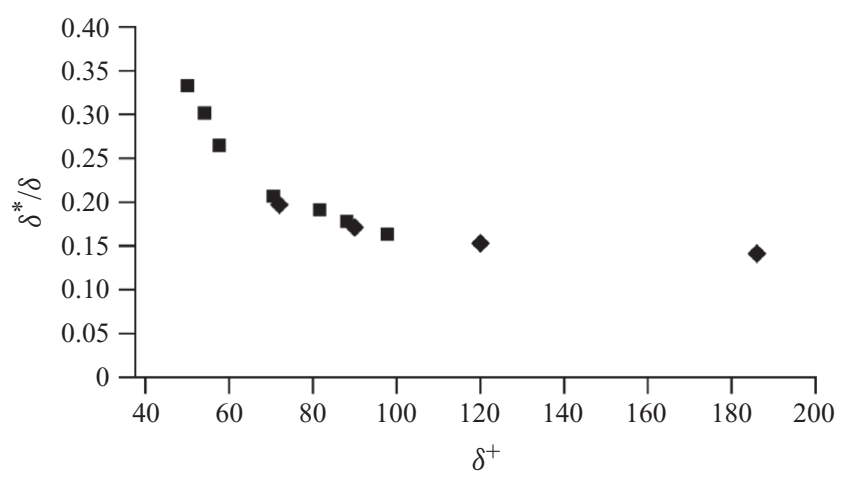

FIGURE 16. Ratio of the displacement thickness to half-channel height computed using (3.4) and plotted versus $\delta^{+}, \mathbf{\square}$, experimental measurements; $\bullet$, DNS data.

Reynolds number the decay rate of these profiles is given by

$$
\frac{\mathrm{d} U^{+}}{\mathrm{d} y^{+}}=\frac{1}{\kappa y^{+}},
$$

where $\kappa \simeq 0.4$ is the von Kármán constant. Consideration of the mean vorticity transport equation (4.1) in concert with (1.4) reveals that the mean vorticity profile admits the same self-similar behaviours as does the Reynolds stress profile. As discussed in $\S 1.3$, these behaviours are responsible for the development of a logarithmic mean velocity profile. The data of figure 15 reveal that through the nonlinear development stage the mean vorticity continues to concentrate toward the wall. Away from the wall, the $\left|\Omega_{z}\right|$ profile develops toward a shape commensurate with a logarithmic mean velocity profile.

These simultaneous processes are given another level of quantification by considering the displacement thickness, $\delta^{*}$, which physically represents the distance from the wall to the centroid of the mean vorticity distribution, i.e.

$$
\delta^{*}=\frac{\int_{0}^{\delta} y \Omega_{z} \mathrm{~d} y}{\int_{0}^{\delta} \Omega_{z} \mathrm{~d} y} .
$$

Figure 16 shows the values of $\delta^{*} / \delta$ computed using (3.4). The profiles being integrated in the numerator and denominator of (3.4) are displayed in figures 15 and 17, respectively. Given the behaviours of these functions with increasing $\delta^{+}$, it is not surprising that the underlying integral curves (not shown) develop an increasingly linear profile. The DNS and experimental data of figure 16 convincingly merge to form a single curve. This curve indicates that, over the given $\delta^{+}$range, the centroid of the mean vorticity distribution moves from $y=\delta / 3$ to $y<\delta / 7$. Consistent with the profiles of figure 15 , this decrease in $\delta^{*} / \delta$ occurs most rapidly prior to, and during, the onset of the nonlinear development stage. The 'knee' of the curve in figure 16 occurs slightly before $\delta^{+}=70$. Figures 17 and 18 provide evidence that logarithmiclike behaviours begin to substantively appear after this initial and rapid redistribution of mean vorticity.

To more directly search for evidence of a developing logarithmic layer, the mean vorticity profile data were weighted with the distance from the wall to form the 


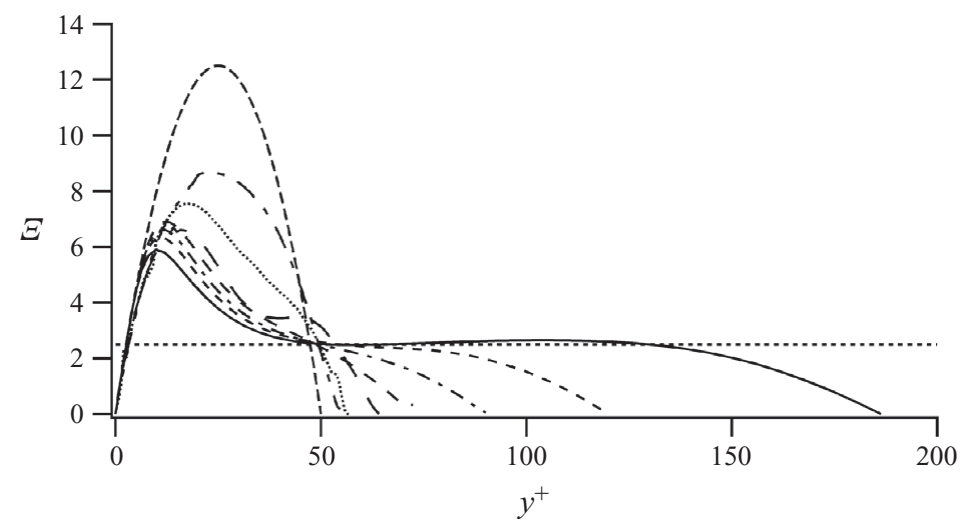

FIGURE 17. Mean velocity profile slope indicator function profiles for varying $\delta^{+}$. Line styles are the same as in figure 15. The dotted horizontal line is at $2.5=1 / 0.4$.

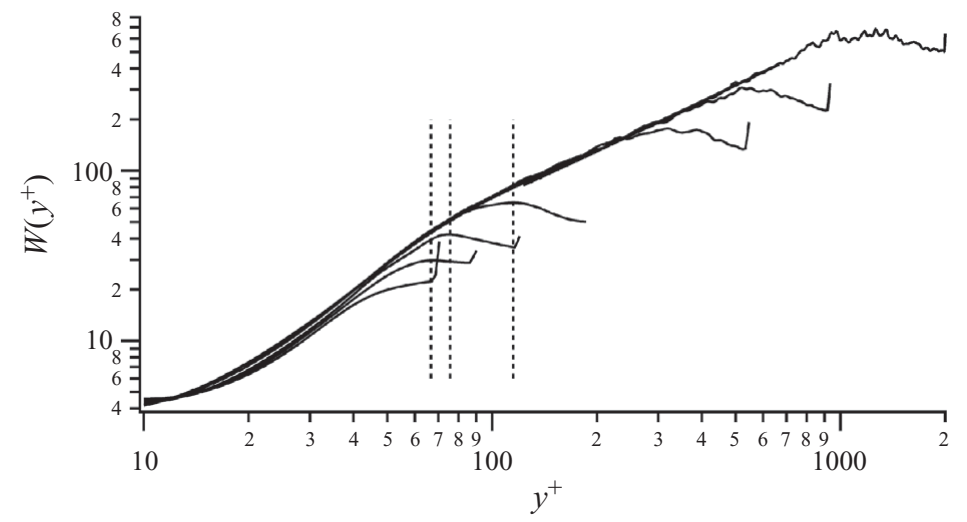

FIGURE 18. Inner-normalized characteristic lengths associated with the continuous hierarchy of scaling layers admitted by the momentum equation for turbulent channel flow (Fife et al. $2005 a$ ). Data are for the DNS studies of Laadhari (2002) at $\delta^{+}=72,90$ and 120 , and Hoyas \& Jimenez (2006) at $\delta^{+}=186,547,934$ and 2003. The curve-fit of the $\delta^{+}=2003$ profile yields $W=0.6247 y^{+}+5.61$ for $118 \leqslant y^{+} \leqslant 667$ (see Klewicki et al. 2009). The vertical dashed lines denote the $\delta^{+}=90,120$ and 186 positions plotted in figure $13(a)$.

indicator function, $\Xi=y^{+} \mathrm{d} U^{+} / \mathrm{d} y^{+}$, profiles of which are plotted in figure 17. This function is often employed in studies of mean profile behaviour, since a zone of logarithmic dependence with slope $\kappa^{-1}$ is revealed where $\Xi=\kappa^{-1}$. This feature is best approximated in figure 17 by the DNS profile at $\delta^{+}=186$. At $\delta^{+}=54.1$ and 57.6, the present data exhibit no evidence of a constant slope region. At $\delta^{+}=70.5$, however, the indicator function exhibits a small but apparent region of approximately constant $y^{+} \mathrm{d} U^{+} / \mathrm{d} y^{+} \simeq 3.2$ between $30 \lesssim y^{+} \lesssim 40$. This region corresponds to a section of approximately constant slope in the $\delta^{+}=70.5$ profile in figure 4 , and also nominally correlates with the region between $y_{m}^{+}$and the centre position of the interior inertial zone shown in figure 11. Plateau-like regions similarly exist in the DNS profiles at $\delta^{+}=90$ and 120 . These plateau-like regions also consistently reside in the region between $y_{m}^{+}$and centre of the inertial peak positions plotted in figure 13(a).

Although the indicator function allows one to quantify logarithmic-like behaviour, it is merely a mathematical tool having no specific relationship to (1.3). Conversely, 
the theory described in $\S 1.3$ reveals the conditions required for (1.3) to admit a logarithmic mean velocity profile solution (Fife et al. 2005a, 2009; Klewicki et al. 2009). These analyses reveal the existence of (approximately) self-similar dynamics on an internal layer hierarchy. A central affiliated attribute is that, on an internal sub-domain of the hierarchy, the layer width distribution, given by (1.6), becomes increasingly linear with increasing $\delta^{+}$.

In $\S 1.3$ we noted that calculations utilizing DNS data at $\delta^{+}$values in the four-layer regime precisely support these analytical predictions. For purposes of comparison, these data are plotted in figure 18. As indicated, the linear curve-fit of the $\delta^{+}=2003$ distribution yields a slope of 0.6247 , or equivalently, specifies that the leading coefficient for the logarithmic equation at this $\delta^{+}$is 0.391 . This independent quantification of the mean profile leading coefficient is essentially identical to the value of $\kappa$ recently estimated via direct measurements of the profile slope (Nagib \& Chauhan 2008).

Figure 18 also presents the distributions of $W$ from the DNS of Laadhari (2002) at lower $\delta^{+}$. Though not entirely clear in figure 18, a close examination of the profiles at $\delta^{+}=90,120$ and 186 reveals that each exhibits a range of $y^{+}$values over which the profile is nominally linear and increasingly parallel to the more well-defined slope of the higher Reynolds number profiles. Comparison with the profiles of figure 4 indicates that approximately logarithmic behaviour correlates with the $y^{+}$range over which the $W$ profiles are approximately linear. Relative to the analysis associated with figure 13 , it is similarly relevant to note that these nascent logarithmic regions consistently exist between $y_{m}^{+}$and the centre position of the inertial zone. Furthermore, as indicated by the dashed vertical lines on figure 18, the position at which $W$ attains its maximum corresponds closely to the measured position of these inertial zones as by definition, they must. (Note that the DNS data at $\delta^{+}=72$ does not exhibit a clear peak in $W$, and correspondingly, does not exhibit evidence of an internal inertial zone.) All of these traits are embraced by the theory outlined in $\S 1.3$. Central to this is the fact that (1.3) admits an invariant form on the $L_{\beta}$ hierarchy. The present results provide evidence that the primary attributes of the functions underlying the characteristic features of the high Reynolds number flows (e.g. distance from the wall scaling, logarithmic mean velocity profile) begin to emerge soon after the onset of the nonlinear development stage, and well before the four-layer structure is established.

\section{Discussion and conclusions}

To the authors' knowledge, the present study is the first to provide a detailed exploration of the mean momentum equation development in non-laminar channel flows for $50 \lesssim \delta^{+} \lesssim 190$. Given this, a primary aim has been to expose how the -1 ratio of the two terms in (1.1) evolves to the structure characterizing (1.2) in the fourlayer regime. Distinctive properties of the latter include a mean momentum balance composed of three terms having an increasingly disparate ordering (sometimes two, sometimes three dominant terms) in the different layers. An overarching theme of the following discussion is on how the present transitional flow observations connect to those in the four-layer regime. This is aided by the theory described in $\S 1.3$.

\subsection{On the initiation of the Reynolds stress profile}

The Reynolds stress gradient is the dynamical ingredient that leads to the layer structure of figure 1. According to linear stability theory, the mean effects of turbulent inertia are first realized near the critical layer of the most unstable disturbance, e.g. 
Criminale et al. (2003). Owing to the nature of these instabilities, the position of the critical layer in parallel shear flow is typically a relatively small distance from the wall (say $y / \delta \simeq 0.1$ ), e.g. Sherman (1990). Based upon the mean profiles of figure 3, one can ascertain that in the present experiments a finite Reynolds stress distribution first appears for Reynolds numbers $50 \lesssim \delta^{+} \lesssim 52$. Therefore, a disturbance spectrum containing the most unstable wavenumber is expected to initially generate a Reynolds stress profile in the region $5 \lesssim y^{+} \lesssim 10$.

Jordinson (1970) numerically solved the Orr-Sommerfeld equation. His formulation employed boundary conditions appropriate to the boundary layer, but assumed parallel streamlines as in the case of fully developed channel flow. Jordinson's calculations show that the Reynolds stresses, $T=-\rho \overline{u v}$, produced by disturbances in a subcritical flow decay owing to a significant zone of negative $T$ in the outer region. As Reynolds numbers exceed the critical value, however, this outer zone of negative $T$ rapidly diminishes such that the overall disturbance field grows. In all of the cases studied by Jordinson, the positive (critical layer) peak in $T$ remained at essentially fixed $y / \delta$, and in his supercritical cases the initial distributions of non-zero $T$ were spatially confined to $y / \delta \leqslant 0.4$. Jordinson's calculations also provide evidence that the width of the positive $T$ distribution resultant from linearly unstable modes increases with increasing Reynolds number.

Finite amplitude fluctuations are a prerequisite to the onset of the nonlinear development stage. As such, it is rational to surmise that the initial set of nonlinear interactions is largely determined by the distribution of linearly unstable modes. That is, within a neighbourhood of the initiating bifurcation sequence (as, for example, plausibly described by Orszag \& Patera 1983), and for sufficiently low $\delta^{+}$, the Reynolds stress distribution based upon the linearly unstable modes is expected to be an accurate representation of the actual flow field realization. At fixed Reynolds numbers above the critical Reynolds number, the actual set of excited linear modes depends on the wavenumber and frequency content of the disturbance field. From this, one can reason that perturbations that excite all of the neutrally stable modes will produce a broader $T$ distribution than those that only excite a narrower band of these modes. Similarly, as the Reynolds number increases, the distribution of potentially excitable linear modes is known to broaden. Jordinson's calculations cited above provide evidence that a fixed disturbance generates an increasingly broader $T$ profile as the Reynolds number increases. Both of these effects are generally expected to influence the shape of the transitional Reynolds stress distribution, and especially at low $\delta^{+}$as the nonlinear interactions relatively quickly dominate the linear modes with increasing $\delta^{+}$.

\subsection{On the observed differences between the results from experiments and DNS}

Most of the figures herein simultaneously present experimental and DNS data. For the more common plots, the quantitative comparison is good, e.g. figures 3,7 and 16. Furthermore, for profiles derived via the differencing of quantities (e.g. figures 5 and 17) the data trends are also highly consistent. For quantities appearing in (1.3), however, the quantitative differences are more evident. Specifically, we cannot conclusively say why the measured Reynolds stress profiles exhibit a more dramatic transition from concave downward to concave upward curvature than do the DNS profiles (see figure 10). This issue is significant, since it pertains to the correspondences between the inertial zones, whose properties are quantified in figures 12 and $13(a)$, and important elements of the theory described in $\S 1.3$. We thus offer some further observations and ideas. 
One observation is that, except for the $\delta^{+}=72$ DNS, all of the DNS and experimental $T^{+}$profiles examined exhibit the qualitative feature of a region (for $y^{+}>y_{m}^{+}$) of concave downward curvature, followed by a region of curvature that is concave upward. The study of Jordinson (1970) shows that this feature also exists in the initial $T$ distributions generated by supercritical linear instabilities. Similarly, the analyses of Klewicki et al. (2009) confirm that this property consistently exists in fully turbulent channel flow up to the highest $\delta^{+}$accessible by DNS $\left(\delta^{+}=2003\right)$. The recent transitional pipe flow experiments of Natrajan \& Christensen $(2007,2009)$ also clearly demonstrate these features, and do so to a degree that is visually more apparent than in the present experiments. These independent observations are significant for a number of reasons: (i) they employed PIV data that directly measured the $T^{+}$ profile, (ii) the pipe flow mean momentum balance can be cast into a form that is identical to that for channel flow, (iii) high Reynolds number pipe flow data exhibit a four-layer structure that is empirically indistinguishable from channel flow (Wei et al. 2005a) and (iv) comparison with the low $\delta^{+}$DNS pipe flow data of Wu \& Moin (2008) reveals a more gradual transition from concave downward to concave upward curvature in the DNS $T^{+}$profile at the same $\delta^{+}$, i.e. exactly what the present comparisons show for channel flow. In addition to these observations, we note that although the shapes of the experimental profiles in figure 10 noticeably vary from the DNS profiles, the positions and values of the inertial peaks (maxima in $\left|\mathrm{d} T^{+} / \mathrm{d} y^{+}\right|$) from the experiments and DNS merge (albeit, not perfectly) to nominally define single curves (see figures 12 and $13 a$ ). Given the evidence at both higher and lower Reynolds numbers than in the present experiments and DNS, it is surmised that these qualitative features pertaining to the curvature of the Reynolds stress profile are robust, but that the detailed quantitative features remain an open question and may vary - especially early in the transitional regime.

A number of factors potentially underlie the observed quantitative differences between the experimental and DNS curves of figure 10. As noted by one referee, this could be associated with the propagation of errors in either the experiments or DNS, or may be associated with secondary flows owing to the finite aspect ratio of the channel. Regarding the accuracy of the MTV data of figure 3, it is pertinent to note that the difference between the $\delta^{+}=52.6$ profile and the analytical laminar flow profile is visually imperceptible. It only becomes apparent that this flow is not laminar after its derivatives are computed and the associated Reynolds stress profile is estimated. Furthermore, there are no a priori reasons to attribute the observed differences to experimental uncertainty - especially in the outer region where the MTV method is expected to perform best. We did, however, also compute the second derivatives of the laminar profiles of Elsnab et al. (2010). These calculations have utility since one can compare with the known analytical solution. The results indicate that the noise in the second derivative is symmetrically and randomly distributed about the mean, and except near the wall, exhibit no apparent bias in the noise amplitude that correlates with the distance from the wall. Across all profiles, the measured error magnitude from the mean was about $12 \%$ (which also includes the uncertainty in the measured pressure gradient), and the standard deviation of the scatter about the mean was about $4 \%$. These observations are relevant since random errors cannot account for why the discrepancies are spatially localized as depicted in figure 10. Similarly, a rather special systematic bias error is required to generate the observed agreement in the clear monotonic trends depicted in figures 12 and 13(a,b). Moreover, while any real channel experiment is susceptible to finite channel width effects, there is no apparent reason to expect that secondary flow effects might also (and remarkably) 
produce the observed $\delta^{+}$trends in figures 12 and 13(a,b). Similar speculations could be made regarding the capacity of DNS to accurately capture low Reynolds number outer layer phenomena. One potential issue here is that, relative to those at high Reynolds number, these flows have elevated viscous forces in the outer region, and thus are likely to produce smaller scale dynamical motions.

In light of the above considerations, it seems at least as plausible that the observed differences are not primarily affiliated with experimental or computational errors, but rather are associated with the initiation of the Reynolds stress distribution as discussed in $\S 4.1$. Specifically, physical channel (or pipe) experiments are realized by a developing flow that eventually attains a fully developed state. The disturbance field generated in these developing flows will non-trivially influence the initial $T$ distribution at the given measurement location. Conversely, DNS attains the fully developed state through the continual recycling of the out-flow. With each recycling, a broader spectrum of fluctuations is introduced into the in-flow. This will generally result in a broadening of the Reynolds stress profile. Owing to this effect, one can rationally surmise that at a fixed $\delta^{+}$the accumulated disturbance field in the DNS will typically be broader than in experiments. This will diminish the occurrence of spatially intermittent patches of turbulence, produce a fuller Reynolds stress profile and thus produce a more gradual transition through the outer region inflection point. With increasing $\delta^{+}$these differences are expected to diminish as the nonlinear interactions become dominant. This explanation, while not conclusive, embraces the present observations.

\subsection{Physical interpretations}

Figure 5 reveals that over the small Reynolds number increment from laminar flow up to $\delta^{+}=54.1$ the $\overline{u v}^{+}$profile develops rapidly, attaining a peak value exceeding 0.2 . Furthermore, the $\delta^{+}=54.1$ datum in figure 6 indicates that $y_{m}^{+}=18.1$. This position is closer to the wall than expected from the trend exhibited by the higher $\delta^{+}$data on that plot, but much farther from the wall than the estimated position of the critical layer. Together, these observations support the conclusion that there is an initial and rapid post-instability stage, followed by flow developments that are increasingly recognizable as the mechanisms of fully turbulent flow. It is further relevant to note that the lowest Reynolds number data $\left(\delta^{+}=52.6\right)$ provide evidence of an internal zone where the mean mechanism of turbulent inertia attains a magnitude comparable to the mean viscous and pressure forces, but that outside this zone the dynamics are dominated by the laminar force balance. Thus, as described in $\S 3.3$, layer III is the first to be realized. From this we surmise that the initial stage is largely associated with the spreading of turbulent inertia such that its influence fills the entire halfchannel. This spreading is rationally expected to occur at an elevated rate until the underlying motions become constrained by the boundaries. Evidence to this effect is given by the relatively rapid variation of $y_{m}^{+}$at low $\delta^{+}$. For $y^{+}>y_{m}^{+}$, the spreading of turbulent inertia is characterized by the outward movement of the inertial zone quantified in figure 13(a), which, even prior to the four-layer regime, was shown to closely correlate with the position of $W_{\max }$ in figure 18 . Interior to $y_{m}^{+}$, the spreading is constrained by the influence of the wall and the amplification of the viscous force also reflected by the wallward concentration of vorticity. Figure 9 shows that both terms $B$ and $C$ in (1.3) settle into their asymptotic peak positions near $y^{+}=7$. At higher $\delta^{+}$, the Reynolds stress distribution continues to develop. Notably, the region around $T_{m}^{+}=-\overline{u v_{m}^{+}}$continues to broaden so as to realize the $\sqrt{\delta^{+}}$scaling behaviours of layers II and III (Fife et al. 2005b; Klewicki et al. 2007; Klewicki 2010). 


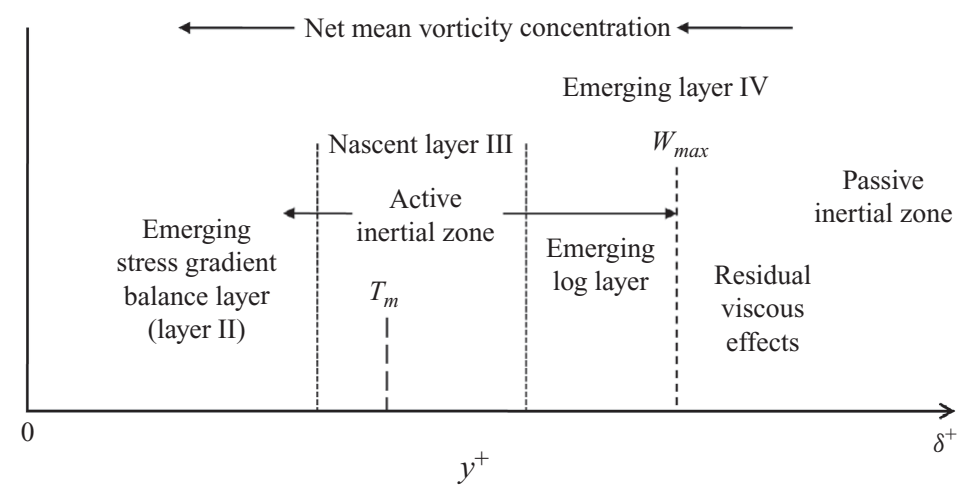

FIGURE 19. Schematic representation of the momentum and vorticity redistribution processes during the nonlinear development stage. The interactions that increasingly dominate during this stage eventually lead to the four-layer structure characteristic of high Reynolds number flow depicted in figure 1 . The arrows denote the relative expansion of the noted regions/influences.

The existence of a peak in the Reynolds stress inherently leads to a non-zero momentum source effect from very near the wall to $y_{m}^{+}$, and a momentum sink effect from $y_{m}^{+}$to $y^{+}=\delta$. The mean vorticity transport equation,

$$
0=\frac{\mathrm{d}^{2} \Omega_{z}^{+}}{\mathrm{d} y^{+2}}+\frac{\mathrm{d}^{2} T^{+}}{\mathrm{d} y^{+2}}
$$

reveals that for all non-laminar $\delta^{+}$the curvature of the $\Omega_{z}^{+}$profile is mirrored by that of $T^{+}$. Consistently, the analysis of (1.3) by Eyink (2008) indicates that the mechanisms underlying the outward flux of vorticity are identical to those responsible for the inward flux of momentum (also see Klewicki et al. 2007). Thus, the action of turbulent inertia leads to the observed spreading of outer region vorticity, along with the attendant reduction in the mean velocity curvature (viscous stress gradient). Evidently, this process gives rise to two regions of inertially dominated flow. One is positioned between $y_{m}^{+}$(or, more precisely, where terms $B$ and $C$ cross) and the peak positions of figure $13(a)$ (or, equivalently, the position of $W_{\max }$ ), and the other is in the vicinity of $\delta$. In the transitional regime, these inertial regions are separated by a continually diminishing zone where the mean viscous force has relatively elevated importance. This structure of a residual viscous zone sandwiched between two inertial regions is exemplified in figure 11. Near $\delta$, the mean flow becomes inertially dominated, at least in part, simply because the mean vorticity and its gradient are increasingly attenuated. Thus, in the schematic representation of figure 19 the two inertial regions are characterized as active and passive, respectively. Of course, as the $T_{m}^{+}$value increases and the mean vorticity continues to concentrate closer to the surface, the outer inertial region increasingly gains its layer IV character, and in doing so its inertial dynamics are also expected to gain significance. The mean viscous force in this region continually attenuates, leaving the layer III and layer IV features characteristic of the four-layer regime (see figure 14).

As depicted in figure 19, inertial dynamics also penetrate wallward from the region surrounding $T_{m}^{+}$. In this case, however, there is an apparent competition between the increasing mean viscous force and the rising Reynolds stress gradient (figure 9). This occurs owing to the inward concentration of velocity profile curvature and the steadily increasing value of $T_{m}^{+}$. As evidenced by figure 14, this process saturates with 
the formation of the stress gradient balance layer (layer II) that is characterized by an increasingly perfect balance between terms $B$ and $C$ in (1.3) with term $A$ being much smaller.

Overall, these observations appear to provide a self-consistent, momentum-equationbased, connection between the nascent turbulent processes that are established following the instability stage (and that emerge through the Reynolds number range considered herein) and those operative in the four-layer regime.

\subsection{Theoretical implications}

The analyses herein provide evidence that the present theoretical framework also mathematically describes the flow development from laminar to fully turbulent flow. This is distinct from more traditional approaches, that generally do not address any specific regime(s) between instability and some poorly defined sufficiently high $\delta^{+}$ value. An essential element of the present analysis is the direct use of the mean momentum equation and the associated quantification of the ordering of the terms in this equation. Namely, the present estimates indicate that in channel flow the fourlayer regime begins at $\delta^{+} \simeq 180$. At lower $\delta^{+}$, the ordering of terms is not distinct. This attribute characterizes the regime between $50 \lesssim \delta^{+} \lesssim 180$.

Previous studies reveal that the ordering associated with the four-layer structure is essential to (1.3) exhibiting its high Reynolds number properties and scaling behaviours. Specifically, the existence of a logarithmic mean profile depends upon the existence of the $L_{\beta}$ hierarchy of scaling layers. This, in turn, depends upon the balance breaking and exchange of forces across each member of the hierarchy, and in particular its average member, layer III of figure 1. The present results provide evidence that layer III forms soon after the onset of the nonlinear development stage, and does so as part of the process by which the functions underlying the properties of the high Reynolds number flow also rapidly develop robust attributes.

The position where terms $B$ and $C$ cross effectively signifies the outer edge of layer III, and thus this attribute of the fully turbulent flow is rapidly established and maintained after the instability stage. The behaviours associated with $W_{\max }$ in the transitional regime were demonstrated to be highly similar to those in the four-layer regime. Once in the four-layer regime, it is known that $W_{\max }$ locates the upper boundary of an internal layer hierarchy upon which the flow asymptotically attains dynamical self-similarity (Fife et al. 2009). Thus, the appearance of this feature directly connects to the development of a logarithmic mean velocity profile - even at these very low Reynolds numbers. The zone between where terms $B$ and $C$ cross and the position of $W_{\max }$ coincides with the position where the indicator function of figure 17 most closely exhibits a plateau-like behaviour, and, consistently, where the length scale distributions of figure 18 most rapidly become linear. While both of these measures are indicative of an approximately logarithmic mean profile, the present theory identifies dynamically significant end points that have direct connection to the solutions admitted by (1.3). As observed previously, these end points also bound the region of the flow where the logarithmic approximation is observed to be most accurately attained at higher Reynolds numbers (Wei et al. 2005a; Nagib \& Chauhan 2008; Klewicki et al. 2009). (Note that neither the indicator function nor the prevalent overlap-layer-based theory provides guidance regarding the bounds on the logarithmic layer, e.g. Smits, McKeon \& Marusic 2011.) Through the nonlinear development stage, the growth rate of the pertinent region is given by the difference in the rate of increase of the curves in figures $13(a)$ and $(b)$, respectively. In accord with the scalings derived 
for the four-layer regime, this difference varies at a rate approaching $\sim \delta^{+}$, as $\delta^{+}$ increases through the transitional regime.

\subsection{Summary}

The present results support the conclusion that turbulent inertia, the mean of which is given by the Reynolds stress gradient, is the driving mechanism of transitional channel flow. This mechanism has its origin in a zone surrounding $T_{m}^{+}$. With increasing Reynolds number the associated inertial dynamics spread both toward the wall and the centreline in accord with the generation of the positive and negative gradients of $T^{+}$interior and exterior to $y_{m}^{+}$, respectively. Significantly, the Reynolds number dependencies associated with these dynamics are rapidly established (at least approximately) post-instability, and thus are inherent properties of both the transitional and four-layer regimes. Beginning with the laminar state, the flow progresses through a stage in which linear instability contributions to the Reynolds stress profile are significant. This nominally corresponds to the abruptly rising portion of the $C_{f}$ curve in figure 7. Nonlinear interactions subsequently underlie the further development of the Reynolds stress gradients, and, as quantified in figures 15 and 16 , lead to the vorticity redistribution processes underlying the emergence of the four-layer structure (figure 14). This first comes into being at $\delta^{+} \simeq 180$.

We thus surmise that the specific dynamical mechanisms initiated owing to a nonzero Reynolds stress gradient distribution are not only responsible for the formation of the four-layer structure, but also in the four-layer regime continue to sustain and more precisely realize the disparate ordering of terms reflected by the structure of figure 1. Lastly, it is relevant to note that the present findings are largely in accord with the perceptive observations of Sreenivasan (1989) and Sreenivasan \& Sahay (1997) that point to the irreducible importance of the dynamics in the vicinity of $T_{m}$ (effectively layer III) and the critical layer origin associated with these dynamics.

This research was supported by NSF IGERT grant DGE 9987616. The efforts of J.K. were also partially supported by the National Science Foundation under grant CBET-0555223, grant monitor, W. Schultz. J.K. also expresses his thanks to Dr J. McHugh for sharing his knowledge regarding linear instabilities. The authors are grateful to all of the referees for their penetrating questions. These led to a significantly better manuscript, and in particular a recognition that the observed position of the inertial zones also identically marks the upper end of the $L_{\beta}$ hierarchy. The authors also thank Dr Hoyas, Dr Jimenez, Dr Kuroda, Dr Kasagi, Dr Hirata and Dr Laadhari for making their DNS data available.

\section{REFERENCES}

Boiko, A., Grek, G., Dovgal, A. \& Kozlov, V. 2002 The Origin of Turbulence in Near-Wall Flows. Springer.

Criminale, W., Jackson, T. \& Joslin, R. 2003 Theory and Computation in Hydrodynamic Stability. Cambridge University Press.

DEAN, R. 1978 Reynolds number dependence of skin friction and other bulk flow variables in two-dimensional rectangular duct flow. J. Fluids Engng 100, 215-223.

Elsnab, J. 2008 Mean velocity profiles in a high aspect ratio microchannel. PhD dissertation, University of Utah, Salt Lake City.

Elsnab, J., Maynes, D., Klewicki, J. \& Ameel, T. 2010 Mean flow structure in high aspect ratio microchannel flows. Exp. Therm. Fluid Sci. 34, 1077-1088.

EyINK, G. 2008 Turbulent flow in pipes and channels as cross-stream 'inverse cascades' of vorticity. Phys. Fluids 20, 125101. 
Fife, P., Wei, T., Klewicki, J. \& McMurtry, P. $2005 a$ Stress gradient balance layers and scale hierarchies in wall bounded turbulent flows. J. Fluid Mech. 532, 165-189.

Fife, P., KleWicki, J., McMurtry, P. \& Wei, T. $2005 b$ Multiscaling in the presence of indeterminacy: Wall-induced turbulence. Multiscale Model. Simul. 4, 936-959.

Fife, P., KLEWICKI \& WeI, T. 2009 Time averaging in turbulence settings may reveal an infinite hierarchy of length scales. J. Discrete Continuous Dyn. Syst. 24, 781-807.

Fukagata, K., Iwamoto, K. \& Kasagi, N. 2002 Contribution of Reynolds stress distribution to the skin friction in wall-bounded flows. Phys. Fluids 14, 173-176.

HiLl, R. \& KLEwiCKI, J. 1996 Data reduction methods for flow tagging velocity measurements. Exp. Fluids 20, 142-152.

Hoyas, S. \& JimeneZ, J. 2006 Scaling the velocity fluctuations in turbulent channels up to $R e_{\tau}=2003$. Phys. Fluids 18, 011702.

JoRDINSON, R. 1970 The flat plate boundary layer. Part 1. Numerical integration of the OrrSommerfeld equation. J. Fluid Mech. 43, 801-811.

Klewicki, J., Fife, P., Wei, T. \& McMurtry, P. 2007 A physical model of the turbulent boundary layer consonant with mean momentum balance structure. Phil. Trans. R. Soc. A 365, 823-839.

Klewicki, J., Fife, P. \& WeI, T. 2009 On the logarithmic mean profile. J. Fluid Mech. 638, 73-93.

KLEWICKI, J. 2010 Reynolds number dependence, scaling, and dynamics of turbulent boundary layers. J. Fluids Engng 132, 091202.

Koochesfahani, M. \& Nocera, D. 2007 Handbook of Experimental Fluid Dynamics, chap. 5.4, Molecular Tagging Velocimetry. Spring.

Kuroda, A., Kasagi, N. \& Hirata, M. 1989 A direct numerical simulation of the fully developed turbulent channel flow. In Proceedings of the International Symposium on Computational Fluid Dynamics, Nagoya, pp. 1174-1179.

LAADHARI, F. 2002 On the evolution of maximum turbulent kinetic energy production in a channel flow. Phys. Fluids 14, L65-L68.

MaYnes, D. \& WebB, A. 2002 Velocity profile characterization in sub-millimeter diameter tubes using molecular tagging velocimetry. Exp. Fluids 32, 3-15.

Metzger, M., Adams, P. \& Fife, P. 2008 Mean momentum balance in moderately favourable pressure gradient turbulent boundary layers. J. Fluid Mech. 617, 107-140.

Moffat, R. 1988 Describing the uncertainties in experimental results. Exp. Therm. Fluid Sci. 1, 3-17.

Moser, R., Kim, J. \& Mansour, N. 1999 Direct numerical simulation of turbulent channel flow up to $R e_{\tau}=590$. Phys. Fluids 11, 943-945.

Nagib, H. \& Chauhan, K. 2008 Variation of von Kármán coefficient in canonical flows. Phys. Fluids 20, 101518.

Natrajan, V. \& Christensen, K. 2007 Microscopic particle image velocimetry measurements of transition to turbulence in microscale capillaries. Exp. Fluids 43 (1), 1-16.

Natrajan, V. \& Christensen, K. 2009 Structural characteristics of transition to turbulence in microscale capillaries. Phys. Fluids 21, 034104.

Orszag, S. \& Kells, L. 1980 Transition to turbulence in plane Poiseuille flow and plane Couette flow. J. Fluid Mech. 96, 159-205.

Orszag, S. \& Patera, A. 1983 Secondary instability of wall-bounded shear flows. J. Fluid Mech. 128, 347-385.

Ponce, A., Wong, P., Way, J. \& Nocera, D. 1993 Intense phosphorescence triggered by alcohols upon formation of a cyclodextrin ternary complex. J. Phys. Chem. 97, 11137-11142.

Shat, R. \& London, A. 1978 Laminar Flow Forced Convection in Ducts. Academic.

Sherman, F. 1990 Viscous Flow. McGraw-Hill.

Smits, A., McKeon, B. \& Marusic, I. 2011 High Reynolds number wall turbulence. Annu. Rev. Fluid Mech. 43, 353-375.

SReEnivasan, K. 1989 The turbulent boundary layer. In Frontiers in Experimental Fluid Mechanics (ed. M. Gad-el-Hak), pp. 159-209. Springer.

Sreenivasan, K. \& Sahay, A. 1997 The persistence of viscous effects in the overlap region and the mean velocity in turbulent pipe and channel flows. In Self-Sustaining Mechanisms of Wall Turbulence (ed. R. Panton), pp. 253-272. Computational Mechanics.

Thurlow, E. \& Klewicki, J. 2000 Experimental study of turbulent Poiseuille-Couette flow. Phys. Fluids. 12, 865-875. 
Wei, T., Fife, P., Klewicki, J. \& McMurtry, P. $2005 a$ Properties of the mean momentum balance in turbulent boundary layer, pipe and channel flows. J. Fluid Mech. 522, 303-327.

Wei, T., McMurtry, P., Klewicki, J. \& Fife, P. 2005b Meso scaling of the Reynolds shear stress in turbulent channel and pipe flows. AIAA J. 43, 2350-2353.

Wei, T., Fife, P., Klewicki, J. \& McMurtry, P. 2005c Scaling heat transfer in fully developed turbulent channel flow. Intl J. Heat Mass Transfer 48, 5284-5296.

WeI, T., Fife, P. \& Klewicki, J. 2007 On scaling the mean momentum balance and its solutions in turbulent Couette-Poiseuille flow. J. Fluid Mech. 573, 371-398.

WU, X. \& Morn, P. 2008 A direct numerical simulation study on the mean velocity characteristics in turbulent pipe flow. J. Fluid Mech. 608, 81-112. 


\section{University Library}

\section{- M M I N E R VA A gateway to Melbourne's research publications}

Minerva Access is the Institutional Repository of The University of Melbourne

Author/s:

Elsnab, J;Klewicki, J;Maynes, D;Ameel, T

Title:

Mean dynamics of transitional channel flow

Date:

2011-07-01

Citation:

Elsnab, J., Klewicki, J., Maynes, D. \& Ameel, T. (2011). Mean dynamics of transitional channel flow. JOURNAL OF FLUID MECHANICS, 678, pp.451-481. https://doi.org/10.1017/ jfm.2011.120.

Publication Status:

Published

Persistent Link:

http://hdl.handle.net/11343/33013 